\title{
microRNA-1 regulates sarcomere formation and suppresses smooth muscle gene expression in the mammalian heart
}

\section{Amy Heidersbach ${ }^{1,2,3}$, Chris Saxby ${ }^{1}$, Karen Carver-Moore ${ }^{1}$, Yu Huang ${ }^{1}$, Yen-Sin Ang ${ }^{1,2,3}$, Pieter J de Jong ${ }^{4}$, Kathryn N Ivey ${ }^{1,2 *}$, Deepak Srivastava ${ }^{1,2,3 *}$}

${ }^{1}$ Gladstone Institute of Cardiovascular Disease, San Francisco, United States; ${ }^{2}$ Department of Pediatrics, University of California, San Francisco, San Francisco, United States; ${ }^{3}$ Department of Biochemistry and Biophysics, University of California, San Francisco, San Francisco, United States; ${ }^{4}$ Children's Hospital Oakland Research Institute, Oakland, United States

Abstract microRNA-1 (miR-1) is an evolutionarily conserved, striated muscle-enriched miRNA. Most mammalian genomes contain two copies of miR-1, and in mice, deletion of a single locus, miR-1-2, causes incompletely penetrant lethality and subtle cardiac defects. Here, we report that deletion of miR-1-1 resulted in a phenotype similar to that of the miR-1-2 mutant. Compound miR-1 knockout mice died uniformly before weaning due to severe cardiac dysfunction. miR-1-null cardiomyocytes had abnormal sarcomere organization and decreased phosphorylation of the regulatory myosin light chain-2 (MLC2), a critical cytoskeletal regulator. The smooth muscle-restricted inhibitor of MLC2 phosphorylation, Telokin, was ectopically expressed in the myocardium, along with other smooth muscle genes. miR-1 repressed Telokin expression through direct targeting and by repressing its transcriptional regulator, Myocardin. Our results reveal that miR-1 is required for postnatal cardiac function and reinforces the striated muscle phenotype by regulating both transcriptional and effector nodes of the smooth muscle gene expression network.

*For correspondence: kivey@ gladstone.ucsf.edu (KNI); dsrivastava@gladstone.ucsf.edu (DS)

Competing interests: See page 20

Funding: See page 20

Received: 02 August 2013

Accepted: 05 October 2013

Published: 19 November 2013

Reviewing editor: Margaret Buckingham, Institut Pasteur, France

(c) Copyright Heidersbach et al. This article is distributed under the terms of the Creative Commons Attribution License, which permits unrestricted use and redistribution provided that the original author and source are credited.

\section{Introduction}

Cardiac gene expression is cooperatively regulated by an intertwined network of transcription factors and microRNAs (miRNAs) (Srivastava, 2006; Cordes and Srivastava, 2009). Perturbations in the activity or expression of factors within this network result in cardiac structural and functional defects in animal models and in humans. Among these, serum response factor (SRF) and Myocardin (Myocd) cooperate to directly regulate the myogenic gene program in both cardiac and smooth muscle (Treisman, 1987; Wang et al., 2001; Chen et al., 2002; Sepulveda et al., 2002 reviewed in Wang and Olson, 2004). These factors transcriptionally regulate numerous miRNAs that, in turn, regulate transcription factors to reinforce specific cellular decisions and behavior. (Kwon et al., 2005; Zhao et al., 2005; Niu et al., 2008; Cordes et al., 2009).

miRNAs are small, $\sim 21$ nucleotide (nt), single-stranded RNAs that negatively regulate the stability and translation of mRNA transcripts. miRNAs target sequences within the $3^{\prime}$ UTRs of mRNA transcripts that are highly complementary to the miRNA seed sequence (nt 2-8) and have imperfect complementarity outside of the seed region (Valencia-Sanchez et al., 2006). Due to the degenerate nature of miRNA:mRNA interactions, a single miRNA may have hundreds of mRNA targets (Bartel, 2009). Often miRNAs target multiple genes in a common pathway, thereby amplifying the effect of an individual miRNA on a given biological process (Fish et al., 2008; Cordes et al., 2009). 
eLife digest MicroRNAs are tiny RNAs that do not encode proteins. Instead, they regulate the expression of genes by preventing protein-encoding messenger RNAs from being translated into protein. MicroRNAs are expressed throughout the body, including the heart, where the most abundant microRNA is called miR-1. This is encoded by two nearly identical genes:

miR-1-1 and miR-1-2.

Mice that lack the miR-1-2 gene have various heart abnormalities, but generally survive because they still produce some miR-1 from their remaining miR-1-1 gene. Now, Heidersbach et al. have generated the first mice that specifically lack both miR-1 genes, and shown that these animals die before weaning.

When viewed under the electron microscope, heart muscle from miR-1 double knockout mice lacks the characteristic 'striped', or striated, appearance of normal heart muscle. Additionally, miR-1 double knockout hearts have some gene expression characteristics more similar to the smooth muscle found in the gut and in the walls of blood vessels. Smooth muscle differs from striated muscle in that it lacks sarcomeres: these are bands of fibrous proteins, such as myosin, that are essential for muscle contraction.

In normal mice, an enzyme called MLCK contributes to the formation and function of sarcomeres by adding phosphate groups to myosin molecules. By contrast, in smooth muscle an enzyme called Telokin promotes phosphate group removal, and thus affects the function of sarcomeres. Heidersbach et al. showed that miR-1 interacts directly with Telokin mRNA to prevent its expression in the heart, and simultaneously represses a protein called Myocardin, which directly activates transcription of Telokin. However, when miR-1 is absent, as in the miR-1 double knockout mice, Telokin is expressed in heart muscle, along with many other genes characteristic of smooth muscle.

As well as improving our understanding of the development and functioning of the heart, these findings should shed new light on the role of microRNAs in maintaining the patterns of gene expression that characterize unique cell fates.

DOI: 10.7554/eLife.01323.002

microRNA-1 (miR-1) is a highly conserved miRNA and its expression is enriched specifically in cardiac and skeletal muscle. In mice, it is expressed in the heart and somites of the developing embryo during myogenic differentiation, beginning around embryonic day (E) 8.5 (Zhao et al., 2005; Liu et al., 2007). The cardiac expression of miR-1 increases during development, with a dramatic rise in the postnatal period. RNA sequencing has revealed that miR-1 is the most abundant miRNA in the adult mouse heart, representing up to $40 \%$ of all miRNA transcripts (Rao et al., 2009).

miR-1 is transcribed as part of a bicistronic cluster with another striated muscle-enriched miRNA, miR-133a. In the genomes of most mammals, a duplication event has occurred resulting in two copies of the miR-1/133a locus, with miR-1-2 and miR-133a-1 on chromosome 18 and miR-1-1 and miR-133a-2 on chromosome 2 of the murine genome (Figure 1-figure supplement 1). Both precursors are transcriptionally regulated by several key myogenic transcription factors, including Myogenin, MYOD, SRF, MYOCD (Kwon et al., 2005; Zhao et al., 2005; Rao, 2006) and MEF2 (Liu et al., 2007). When processed, both the miR-1-2 and miR-1-1 precursors give rise to identical mature miR-1 species, suggesting evolutionary pressure on both alleles (Figure 1-figure supplement 2). An additional miRNA cluster encoding miR-133b and miR-206 is expressed uniquely in skeletal muscle, with the mature sequence of miR-206 sharing a common seed with miR-1, but varying by 4 nts outside of the seed region.

Deletion of miR-1-2 in mice (Zhao et alo, 2007), which reduces the total expression of cardiac miR-1 by roughly $50 \%$, results in a spectrum of cardiac defects on a pure 129 background, including incompletely penetrant lethality, cardiomyocyte proliferative defects, and electrophysiological abnormalities. In flies, loss of the single miR-1 gene results in abnormal myogenic differentiation and cell polarity defects in cardiac progenitors (Kwon et al., 2005; Sokol, 2005; King et al., 2011); however, the consequences of complete loss of miR-1 in mammals are unknown.

In this study, we report that targeted deletion of the miR-1-1 locus results in a phenotype similar to that described for miR-1-2 null mice, and that the complete loss of miR-1 is uniformly lethal before weaning due to cardiac dysfunction. We show that the loss of miR-1 results in perinatal heart failure with myocardial sarcomeric defects, hypophosphorylation of Myosin Light Chain 2, and ectopic expression of Telokin, a smooth muscle-restricted inhibitor of Myosin Light Chain 2 phosphorylation. Furthermore, 
we found the SRF co-factor, MYOCD, which is critical for transcriptional activation of both the cardiac and smooth muscle gene programs in vivo (Li et al., 2003; Hoofnagle et al., 2011; Wang et al., 2001; Chen et al., 2002), is directly targeted by miR-1. The smooth muscle isoform of Myocd was preferentially upregulated in the absence of miR-1 and likely contributed to ectopic activation of the smooth muscle gene program in the heart. Our findings reveal that miR-1 is embedded in an SRF-dependent cardiac gene program that promotes sarcomerogenesis and myogenic differentiation, while simultaneously repressing the smooth muscle program.

\section{Results}

\section{Generation and characterization of miR-1-1 null mice}

We used homologous recombination to delete one allele of the miR-1-1 precursor in embryonic stem (ES) cells, with a floxed neomycin cassette used for positive selection. (Figure 1-figure supplement 3 and 'Materials and methods'). Injection of targeted ES cells into blastocysts resulted in high-percentage chimeras that transmitted the targeted allele through the germline. Intercrosses of miR-1-1 heterozygous mice revealed that approximately half of all miR-1-1 homozygous-null mice died before weaning when bred onto a pure 129 strain, similar to miR-1-2 null mice (Figure 1A, upper). This lethality was strain dependent, as miR-1-1 null animals on a mixed background (129/BL6) survived at normal Mendelian ratios until weaning. (Figure 1A, lower). By quantitative RT-PCR (qPCR), we found that total cardiac miR-1 levels were decreased in miR-1-1 knockout animals by about $40 \%$ at postnatal day (P) 2 (Figure 1B).

To evaluate cardiac function, we performed echocardiography on adult miR-1-1 null or wild-type littermates. We found a reduction in fractional shortening, as well as an increase in left ventricular enddiastolic and end-systolic dimension in the miR-1-1 knockout animals, indicating ventricular dilation (Figure 1C). Mild ventricular dilation was confirmed histologically (Figure 1D I and II). Additionally, we observed areas of fibrosis in the ventricular myocardium of the miR-1-1 knockouts (Figure 1D III and IV). Like miR-1-2 knockouts, miR-1-1 knockout animals exhibited subtle conduction abnormalities, including prolonged ventricular depolarization and repolarization, indicated by a broader QRS complex and longer QT interval than controls. Additionally, miR-1-1 knockout mice showed broader P waves without alteration of the PR interval (Figure 1E, Figure 1-figure supplement 4). Intermittent atrial arrhythmias were observed in two of five knockout animals analyzed, but were not observed in wildtype animals (Figure 1-figure supplement 5). Thus, mice lacking miR-1-1 were grossly similar to those lacking miR-1-2, in that they exhibited partial lethality as well as subtle conduction abnormalities.

Cardiac conduction defects in the miR-1-2 knockout mice were at least partially ascribed to dysregulation of the miR-1 target, Irx5. Similarly, we found that Irx5 was upregulated in the miR-1-1 knockout mice (Figure 1-figure supplement 6). miR-1-1 knockout mice also had a partial decrease in mature miR-133a levels (Figure 1B). Previously, using semi-quantitative RT-PCR, the miR-133a precursor levels were reported to be unchanged in the miR-1-2 knockout animals. In this study, the analysis of the mature species by qPCR revealed a slight decrease in miR-133a in weaning-age miR-1-2 null hearts, although not statistically significant (Figure 1-figure supplement 7). Importantly, miR-133a expression was maintained at a level described to be inconsequential in previous reports (Liu et al., 2008).

\section{Generation and characterization of compound miR-1 knockout mice}

To investigate the consequences of complete loss of miR-1, we intercrossed miR-1-1 and miR-1-2 mutant mice (Zhao et al., 2007) to generate double-heterozygous mice in a 129/BL6 mixed background. At weaning, no lethality was observed in the double-heterozygous mice (Figure 2-figure supplement 1) similar to the miR-1-1 knockout on a mixed background. Using gene expression microarray analyses, we found 201 genes that were dysregulated in the single knockouts or double-heterozygous mice (Figure 2-figure supplement 2). Of those, 24 genes were coordinately dysregulated in animals of all three genotypes. The majority of genes (195/201) were similarly altered between the double heterozygotes and at least one of the single knockouts. There were, however, some differences in gene expression between these groups, which may suggest minor functional differences of the miR-1 loci.

Double-heterozygous mice were subsequently intercrossed to generate knockout animals with only

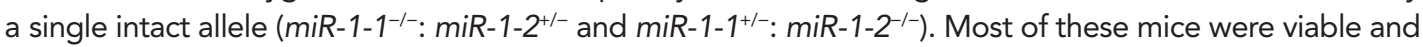
fertile on the mixed background, though mice lacking both the copies of miR-1-2 were under-represented at weaning (Figure 2-figure supplement 3), indicating some difference in the compensatory ability of the two loci. 
A

\begin{tabular}{|c|c|c|c|}
\hline \multirow{3}{*}{$\begin{array}{r}\text { **Observed } \\
\text { Expected }\end{array}$} & $\operatorname{miR}-1-1^{+/+}$ & miR-1-1+/- & miR-1-1-I- \\
\hline & $31(37 \%)$ & $43(50 \%)$ & $11(13 \%)$ \\
\hline & $21(25 \%)$ & $43(50 \%)$ & $21(25 \%)$ \\
\hline
\end{tabular}

Weaning Mixed Strain miR $-1-1^{+/-} \times$miR-1-1 1 -

\begin{tabular}{c|c|c|c|}
\cline { 2 - 4 } & miR-1-1 $^{+/+}$ & miR-1-1+- & miR-1-1-1- \\
\cline { 2 - 4 } $\begin{array}{l}\text { Observed } \\
\text { Expected }\end{array}$ & $23(22 \%)$ & $54(52 \%)$ & $26(25 \%)$ \\
\cline { 2 - 4 } & $26(25 \%)$ & $52(50 \%)$ & $26(25 \%)$ \\
\cline { 2 - 4 } & \multicolumn{3}{|c|}{$\mathbf{n = 1 0 3}$}
\end{tabular}

C
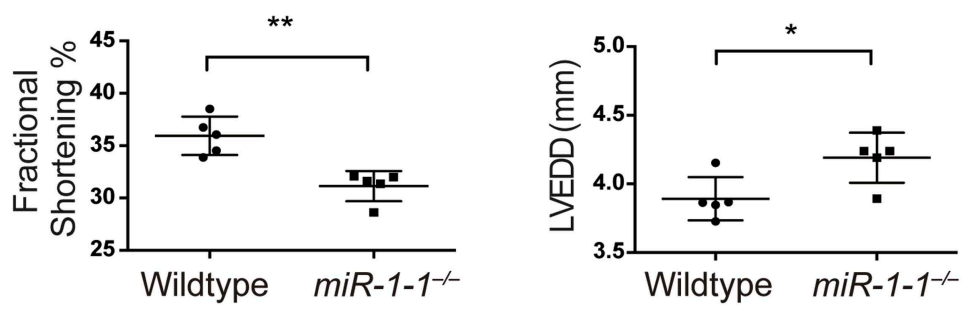

B

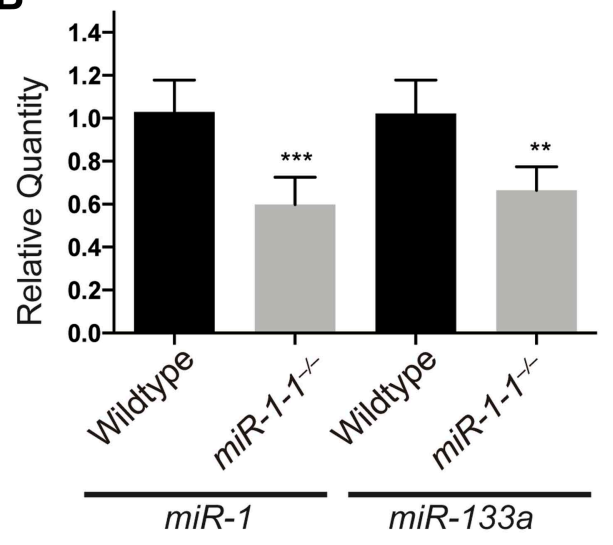

D

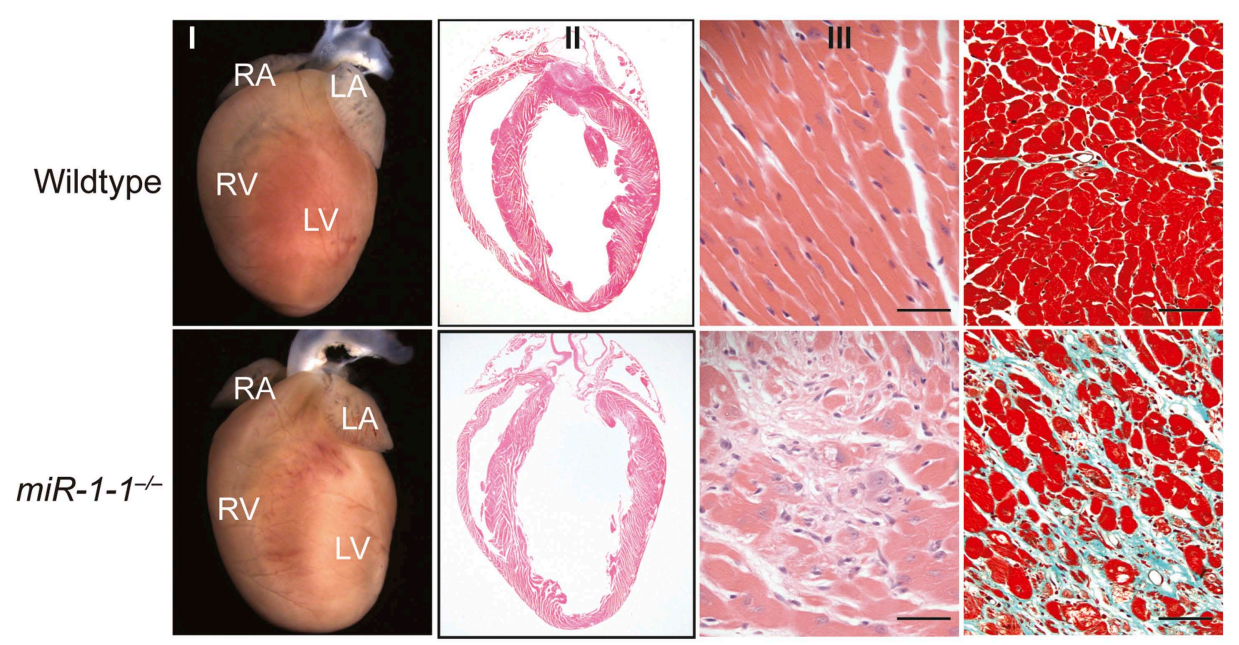

E

\begin{tabular}{c|c|l|l} 
Parameter & Wildtype $(\mathbf{n = 5})$ & $\mathbf{m i R}_{\mathbf{1}-\mathbf{1}^{-/-}} \mathbf{( n = 5 )}$ & $\mathbf{p ~ V a l u e}$ \\
\hline Heart Rate (bpm) & $470 \pm 19$ & $451 \pm 18$ & 0.4744 \\
PR Interval (msec) & $39.9 \pm 1.1$ & $40.6 \pm 1.3$ & 0.6903 \\
P Duration (msec) & $13.1 \pm 0.5$ & $15.9 \pm 0.6^{* *}$ & 0.0077 \\
QRS Interval (msec) & $12.5 \pm 0.5$ & $14.7 \pm 0.3^{* *}$ & 0.003 \\
QT Interval (msec) & $55 \pm 1.1$ & $61 \pm 1.2^{* *}$ & 0.0056 \\
QTc & $49 \pm 0.7$ & $53 \pm 0.5^{* *}$ & 0.0016
\end{tabular}

Figure 1. Viability and cardiac function of miR-1-1-/- mice. (A) Genotypes of offspring generated from miR-1-1+/- intercrosses on either a pure 129 background (upper) or a mixed BL6/129 strain (lower). Numbers of expected and observed genotype ratios are given for weaning-age (3-week-old) pups. (B) qPCR of mature miR-1 and miR-133a in post-natal day 2 hearts. $\mathrm{N}=6$ per genotype. (C) Analyses of cardiac function by echocardiography of Figure 1. Continued on next page 
Figure 1. Continued

adult animals of indicated genotypes on a pure 129 background. $N=5$ per genotype. LVEDD, left ventricular end-diastolic dimension; LVESD, left ventricular end-systolic dimension. (D) Adult wildtype (upper) and miR-1-1-1- (lower) hearts on a pure 129 background (I). RA, right atrium; LA, left atrium; $\mathrm{RV}$, right ventricle; LV, left ventricle. Hematoxylin and eosin images taken at 1.25X magnification (II); and 40X magnification; scale bar indicates $25 \mu \mathrm{m}$ (III). Masson trichrome stain of miR-1-1 knockout myocardium, images taken at 40X; scale bar $50 \mu \mathrm{m}$ (IV) (E) Analyses of cardiac conduction by electrocardiogram (EKG) of adult animals of indicated genotypes on a pure 129 background. ${ }^{*} p<0.05$; ${ }^{* \star} p<0.01$; ns, not significant.

DOI: 10.7554/eLife.01323.003

The following figure supplements are available for figure 1:

Figure supplement 1. Schematic of the miR-1/133a genomic loci.

DOI: 10.7554/eLife.01323.004

Figure supplement 2. Identical sequences of mature miR-1-1 and miR-1-2.

DOI: 10.7554/eLife.01323.005

Figure supplement 3. Left, targeting scheme for deletion of the miR-1-1 locus. DOI: 10.7554/eLife.01323.006

Figure supplement 4. Averaged electrocardiogram tracings tracing from lead I of an adult wild-type or miR-1-1 knockout animal on a pure 129 background.

DOI: 10.7554/eLife.01323.007

Figure supplement 5. Electrocardiogram tracings of an adult wild-type or miR-1-1 knockout animal on a pure 129 background. DOI: 10.7554/eLife.01323.008

Figure supplement 6. qPCR for the miR-1 target, Irx5, in adult miR-1-1-1- and wild-type hearts. DOI: 10.7554/eLife.01323.009

Figure supplement 7. qPCR for mature miR-133a in adult wild-type and miR-1-2 knockout hearts. DOI: 10.7554/eLife.01323.010

Single-allele mice ( $3 / 4$ alleles knocked-out) were intercrossed to generate mice completely lacking miR-1. miR-1 compound-null mice on a mixed background were born at slightly less than Mendelian ratios and were of normal birth weight (Figure 2A, Figure 2-figure supplements 4 and 5). Roughly a quarter of the double-knockout animals died very soon after birth. In a subset of these animals, we observed ventricular septal defects (VSDs) and misalignment of the aorta over the ventricular septum (overriding aorta), likely accounting for their lethality (Figure 2-figure supplement 6). Surviving miR-1 double-knockouts failed to thrive post-natally, with no doubleknockout animals surviving beyond P10 (Figure 2B, Figure 2-figure supplement 5). Examination of surviving miR-1 double-knockouts revealed expansion of the superior portion of the right ventricle (conus) and enlargement of the atria, when compared to wild-type mice beginning at PO (Figure 2C, asterisk). By P4, and more extensively by P10, the dilation of all cardiac chambers was observed, with a particularly notable enlargement of the right atria. Echocardiography revealed severely impaired fractional shortening by P2 (Figure 2D and E) with poor systolic function. Frequent ventricular thrombi were observed by P4, consistent with impaired cardiac function in these animals (Figure 2C). Functional analysis of the conduction system by electrocardiogram (EKG) revealed a spectrum of abnormalities, including a prolonged QRS complex, and prolonged PR and QT intervals (Figure 2F, Figure 2-figure supplement 7). The presence of frequent sinus pauses was observed in all knockout animals analyzed (Figure 2-figure supplement 8). These morphological, functional, and electrophysiological data indicate that the postnatal lethality in miR-1 knockout animals is due to cardiac dysfunction.

qPCR confirmed that miR-1 was not detectable in hearts of miR-1 compound-null mice. Notably, miR-133a expression was decreased in miR-1 double-knockout animals with the dysregulation of miR$133 a$ becoming more pronounced with age as the locus normally becomes more actively transcribed (Figure 2-figure supplement 9). As miR-1 lies transcriptionally upstream of miR-133a, we cannot exclude the possibility that the decrease in miR-133a expression is in part due to impaired transcriptional read-through downstream of the miR-1 targeting event. However, chromatin immunoprecipitation revealed reduced RNA polymerase II (Pol II) occupancy at the miR-1-1/miR-133a-2, and miR-1-2/miR133a-1 promoters in P2 miR-1 double-knockout mouse hearts, suggesting that transcriptional initiation of these loci is reduced secondary to loss of miR-1 expression (Figure 2-figure supplement 10). This suggests a feedback mechanism whereby miR-1 maintains appropriate expression of the miR1/133a loci. 
A

\begin{tabular}{|c|c|c|c|c|}
\hline \multirow{3}{*}{ P0 } & & $\begin{array}{l}\text { miR-1-1-1- } \\
\text { miR-1-1-2- }\end{array}$ & Percentage & Total mice \\
\hline & Observed & 19 (5 dead) & $17 \%$ & 109 \\
\hline & Expected & 27 & $25 \%$ & \\
\hline & Observed & 0 & $0 \%$ & 156 \\
\hline anIng & Expected & 39 & $25 \%$ & \\
\hline
\end{tabular}

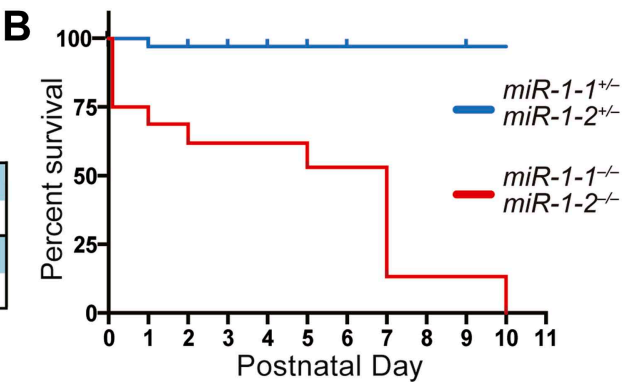

C

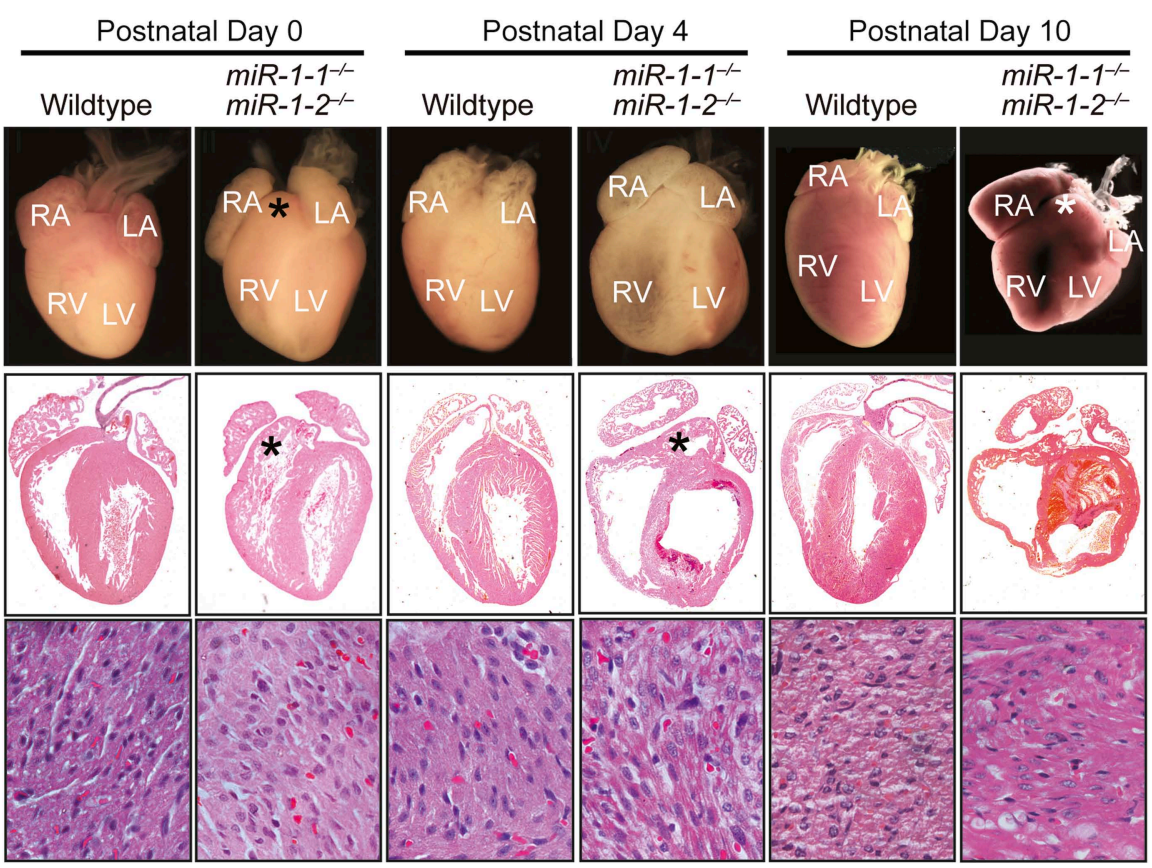

D
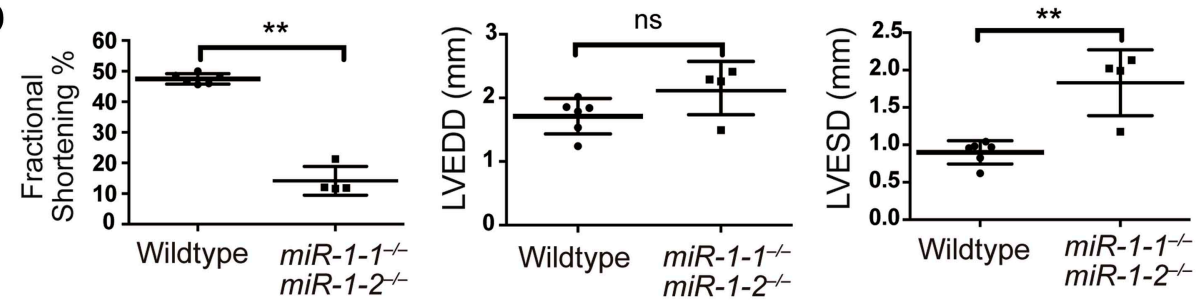

E

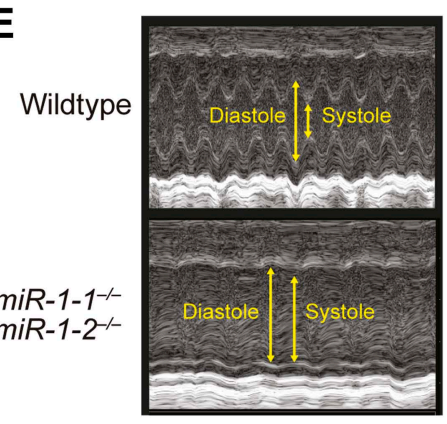

$\mathbf{F}$

\begin{tabular}{c|c|l|r} 
Parameter & Wildtype $(\mathrm{n}=7)$ & $\begin{array}{l}\text { miR-1-1- } \\
\text { miR-1- }-2^{--}(\mathrm{n}=3)\end{array}$ & $\mathrm{p}$ Value \\
\hline Heart Rate (bpm) & $591 \pm 4$ & $398 \pm 21^{* * * *}$ & $<0.0001$ \\
PR Interval (msec) & $33.9 \pm 0.3$ & $39.0 \pm 2.5^{*}$ & 0.0124 \\
P Duration (msec) & $9.0 \pm 0.2$ & $19.4 \pm 1.9^{* * * *}$ & $<0.0001$ \\
QRS Interval (msec) & $8.0 \pm 0.2$ & $14.6 \pm 0.8^{* * * *}$ & $<0.0001$ \\
QT Interval (msec) & $45 \pm 0.6$ & $68 \pm 2.1^{* * * *}$ & $<0.0001$ \\
QTc & $44 \pm 0.6$ & $55 \pm 0.2^{* * * *}$ & $<0.0001$
\end{tabular}

Figure 2. Compound miR-1 knockout mice exhibit lethality due to a spectrum of cardiac defects. (A) Genotypes of offspring generated from

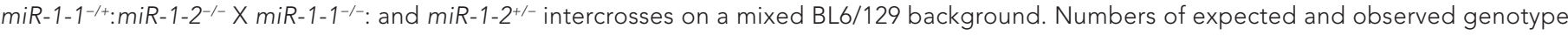
ratios are given for post-natal day 0 (P0) and weaning-age (3-week-old) pups. (B) Kaplan-Meier survival curve of miR-1 double-knockout animals and Figure 2. Continued on next page 
Figure 2. Continued

double-heterozygous littermates. (C) Abnormal cardiac morphology in postnatal miR-1 double-knockout mice includes an elongated outflow tract, as evidenced by bulging of the conus at PO (asterisk). By P4, chamber dilation and thinning of the myocardium was apparent and ventricular clots were commonly observed. RA, right atrium; LA, left atrium; RV, right ventricle; LV, left ventricle. Middle panel images taken at 1.25X magnification; lower panel images taken at 40X magnification. (D) Echocardiography showed abnormal cardiac function in P2 miR-1 null compared to wild-type animals. $\mathrm{N}=7$ for wild-type mice and $\mathrm{N}=4$ for miR-1 null mice. LVEDD, left ventricular end-diastolic dimension; LVESD, left ventricular end-systolic dimension. (E) Representative M-mode image by echocardiography indicating diastolic and systolic dimensions. (F) Electrocardiographic analysis revealed conduction abnormalities in miR-1 double-knockout animals by P2, including a decreased heart rate and elongated QRS relative to wild-type controls. ${ }^{*} \mathrm{p}<0.05$; ${ }^{* *} \mathrm{p}<0.01 ;{ }^{* * *} \mathrm{p}<0.001 ;{ }^{* * * *} \mathrm{p}<0.0001 ; \mathrm{ns}$, not significant.

DOI: 10.7554/eLife.01323.011

The following figure supplements are available for figure 2:

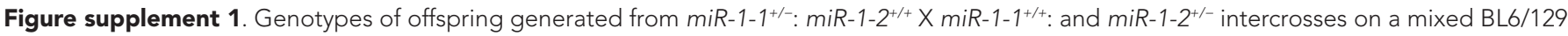
background.

DOI: 10.7554/eLife.01323.012

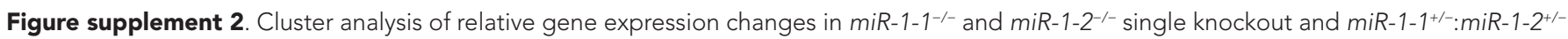
double-heterozygous hearts.

DOl: 10.7554/eLife.01323.013

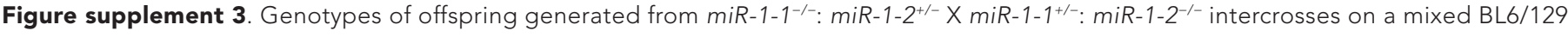
background at weaning.

DOI: 10.7554/eLife.01323.014

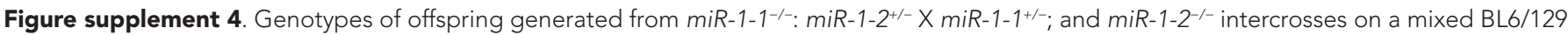
background at birth.

DOI: 10.7554/eLife.01323.015

Figure supplement 5. miR-1 double-knockouts fail to thrive.

DOI: 10.7554/eLife.01323.016

Figure supplement 6. Abnormal cardiac morphology in miR-1 double-knockouts found dead at birth compared to wild-type control. DOI: 10.7554/eLife.01323.017

Figure supplement 7. Averaged electrocardiogram tracings from lead I of P2 miR-1 null (right) or wild-type (left) mice. DOI: 10.7554/eLife.01323.018

Figure supplement 8. Electrocardiogram tracings of a postnatal wild-type or miR-1 null animal on a mixed background. DOI: 10.7554/eLife.01323.019

Figure supplement 9. Left, qPCR of mature miR-1 or miR-133a in E12.5 wild-type or miR-1 null hearts ( $\mathrm{n}=3$ per genotype). Right, mature miRNA expression in $\mathrm{PO}$ wild-type or miR-1 null hearts ( $\mathrm{n}=5$ per genotype).

DOI: 10.7554/eLife.01323.020

Figure supplement 10. qPCR to detect the miR-1-2/133a-1 or miR-1-1/133a-2 promoter sequences, or an intergenic genomic sequence, following chromatin immunoprecipitation (ChIP) of RNA polymerase II (RNA Pol II) in P2 wild-type or miR-1 null hearts.

DOI: 10.7554/eLife.01323.021

\section{Cardiomyocytes lacking miR-1 show sarcomeric defects}

Given the relatively normal cardiac morphogenesis in surviving miR-1 knockouts, we hypothesized that their impaired cardiac contractility was due to a primary myocardial defect. In agreement, transmission electron microscopy of ventricular tissue revealed the areas of extensive sarcomeric disruption at P0, before the onset of ventricular dilation (Figure 3A). Mitochondrial morphology was also abnormal, including overall decreased mitochondrial size (Figure 3-figure supplements 1 and 2) and decreased complexity of mitochondrial cristae (Figure 3-figure supplement 1).

Sarcomeric morphology was further analyzed in isolated PO neonatal cardiomyocytes by immunostaining for the Z-line protein a-Actinin, as well as by Phalloidin staining to visualize the filamentous actin cytoskeleton. Individual cardiomyocytes were classified on a scale from I-V based on their sarcomeric organization, with class-I cells showing highly ordered sarcomeres, and class-V cells having no sarcomeric organization (Figure 3-figure supplement 3). Sarcomeres in miR-1 null cardiomyocytes showed significant disruption when compared to those from miR-1 double-heterozygous littermates. Only $10 \%$ of double-knockout cardiomyocytes had highly organized sarcomeres, compared to $45 \%$ of miR-1 double-heterozygous cells. The degree of sarcomeric organization correlated with miR-1 dosage as cardiomyocytes lacking three out of four copies of miR-1 showed an intermediate degree of organization (Figure 3C). 


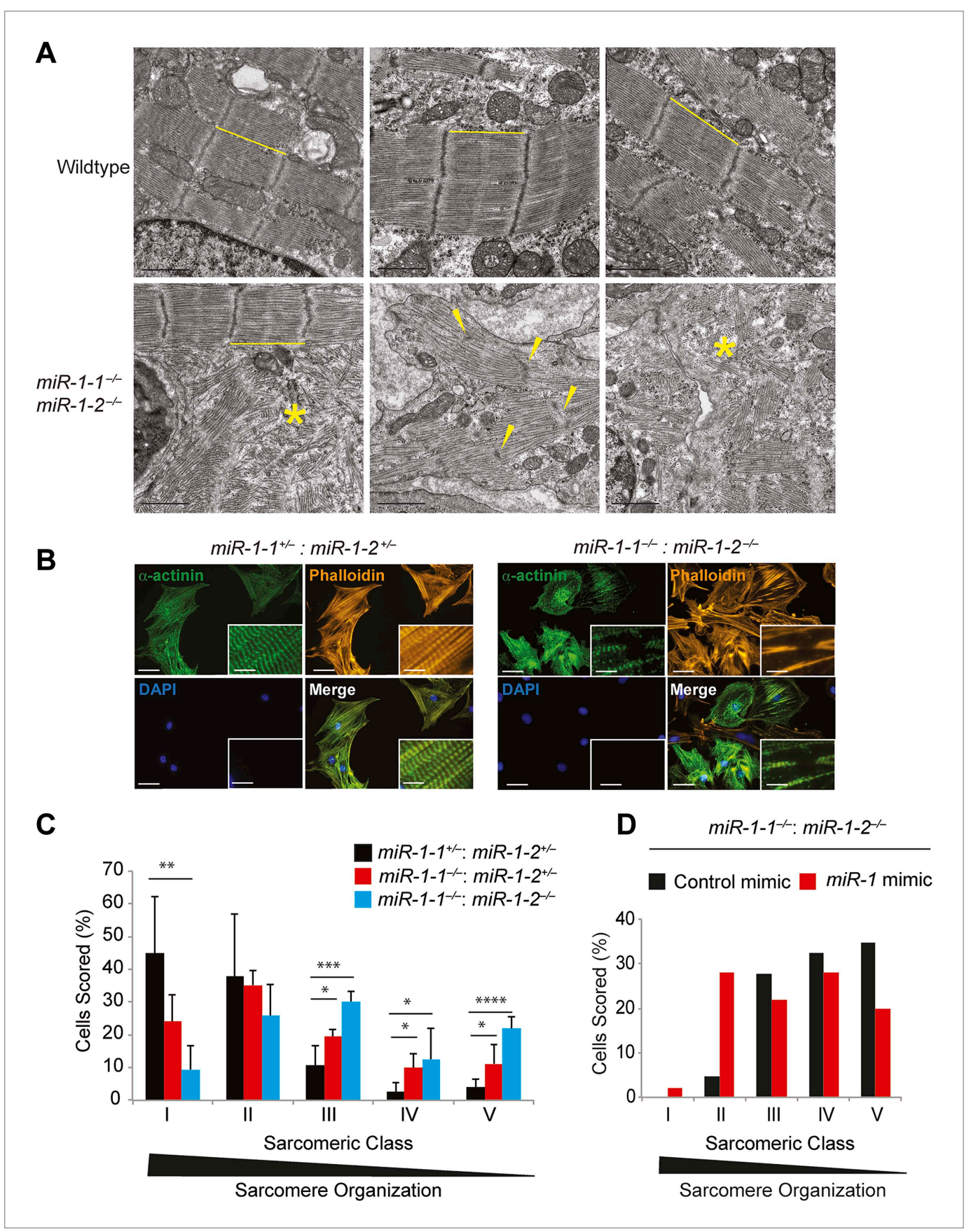

Figure 3. Sarcomere disruption in miR-1 null cardiomyocytes. (A) Transmission electron microscopy (TEM) of PO wild-type or miR-1 double-knockout myocardium. Representative areas of sarcomeric disarray are indicated $\left(^{*}\right)$. Distance between Z-lines is indicated with lines. Arrows indicate disrupted Z-line structures. Scale bar, $1 \mu \mathrm{m}$. (B) Immunofluorescence of sarcomeric structures in isolated PO cardiomyocytes with Phalloidin (F-actin cytoskeleton [orange]) and sarcomeric alpha-actinin (green) DAPI (blue) indicates nuclei. Images captured at 40X magnification. (C) Percentage of cardiomyocytes of individual sarcomeric classes observed. $\mathrm{N}=3$ for miR-1 null mice; $\mathrm{N}=2$ for miR-1-1-1-:miR-1-2+/- mice; and $\mathrm{n}=6$ for miR-1 double-heterozygous mice, with a minimum of 50 cells classified per animal. (D) Representative analysis showing percentage of P5 miR-1 null cardiomyocytes of individual sarcomeric classes observed $24 \mathrm{hr}$ after transfection of a miR-1 mimic or control RNA mimic. Roughly 50 cardiomyocytes per condition were evaluated. ${ }^{*} p<0.05 ;{ }^{* \star} p<0.01 ;{ }^{* \star *} p<0.001 ;{ }^{* \star \star *} p<0.0001$; ns, not significant.

DOI: 10.7554/eLife.01323.022

The following figure supplements are available for figure 3:

Figure 3. Continued on next page 
Figure 3. Continued

Figure supplement 1. Transmission electron microscopy (TEM) reveals mitochondrial morphology defects in miR-1 null hearts.

DOI: 10.7554/eLife.01323.023

Figure supplement 2. Quantification of mitochondrial area from TEM images reveals a reduction in mitochondrial area in both miR-1 double-knockouts compared to controls, although the degree to which mitochondrial area is reduced varies.

DOI: 10.7554/eLife.01323.024

Figure supplement 3. Sarcomeric organization classification scheme.

DOI: 10.7554/eLife.01323.025

To determine if reintroduction of miR-1 was sufficient to improve sarcomere organization in cardiomyocytes from post-natal hearts, we transiently transfected a miR-1 RNA mimic into cultured cardiomyocytes isolated from P5 animals. Indeed, adding miR-1 partially rescued this phenotype and enhanced sarcomeric organization in miR-1 double-knockout cardiomyocytes, compared to those treated with a control RNA mimic (Figure 3D).

\section{Dysregulation of Telokin and myosin light chain phosphorylation}

We performed RNA sequencing of late embryonic stage (E18) miR-1 null and wild-type hearts to identify genes that were dysregulated in the absence of miR-1. We selected this time point in order to reveal primary changes in gene expression due to the loss of miR-1 and not those that may arise secondary to heart failure. Given that many direct miRNA targets are upregulated at the protein, but not transcript level, we expected that sequencing analysis of this stage would identify pathways that are dysregulated in the miR-1 knockout, some of which may involve direct miR-1 targets. We utilized the GREAT interface (McLean et al., 2010) to evaluate the enrichment of miRNA targets within the set of genes that were upregulated in the miR-1 null hearts, compared to wild-type controls (Figure 4figure supplement 1). We found that genes containing miR-1/206 seed sequence complementarity were most significantly enriched in this data set. Genes targeted by miR-495, miR-518a-2, miR-501 and miR-409 were also enriched, though to a lesser degree. Notably, mRNAs with seed sequence complementarity to miR-133a were not enriched, suggesting that the reduction in miR-133a levels did not reach the threshold for significant dysregulation of genes in the miR-1 knockout.

We next performed a gene ontology analysis using the GO-Elite interface (Zambon et al., 2012) to determine at a functional level which dysregulated genes may be phenotypically relevant. We found that genes participating in metabolic and mitochondria-related pathways were downregulated in miR1 knockout, compared to wild-type hearts, consistent with the morphological abnormalities visualized by electron microscopy (Figure 4A). Interestingly, many upregulated genes fell into the 'regulation of actin cytoskeletal' pathway (Figure 4B). As the dysregulation of actins and other cytoskeletal genes contribute to cardiomyopathies, in a complementary analysis, we investigated if any of the upregulated cytoskeletal genes were direct miR-1 targets. miRNA targets remain challenging to predict computationally; therefore, we utilized three different target prediction algorithms-Targetscan (www.targetscan.org), PITA (http://genie.weizmann.ac.il/pubs/mir07/mir07_dyn_data.html), and Pictar (http://pictar.mdcberlin.de/cgi-bin/PicTar_vertebrate.cgi)—to identify consensus predicted targets (Figure 4C, Figure 4source data 1). Of the genes that were expressed at an equal or higher level in the knockout compared to wild-type hearts, 89 genes were predicted miR-1 targets by all three algorithms. Of these, 13 genes were significantly upregulated at the mRNA level in the miR-1 null hearts. (Figure 4C, Figure 4source data 1).

In the miR-1 null animals, myosin light chain kinase (MLCK) was a putative miR-1 target of particular interest, as it was highly upregulated and had a known role in regulating the cytoskeleton (Figure 4B,C). MLCK, which is encoded within the Mylk locus, regulates the cytoskeleton by phosphorylating the regulatory Myosin Light Chain 2 (MLC2). MLC2 is associated with Myosin Heavy Chain (MHC) and is situated adjacent to the actin interaction domain of the globular myosin head. In smooth muscle, MLC2 phosphorylation is sufficient to induce contraction, and in the striated muscle of the heart, phosphorylation increases the rate and magnitude of contractile force (Davis et al., 2001, reviewed in Kamm and Stull (2001)).

The 31 exons of the Mylk locus give rise to four distinct but overlapping gene products (Figure 5A). Two 220-kD MLCK isoforms, which vary only by alternate inclusion of exon 1, are expressed in non-muscle 
A ElectronTransport Chain: Oxidative phosphorylation:

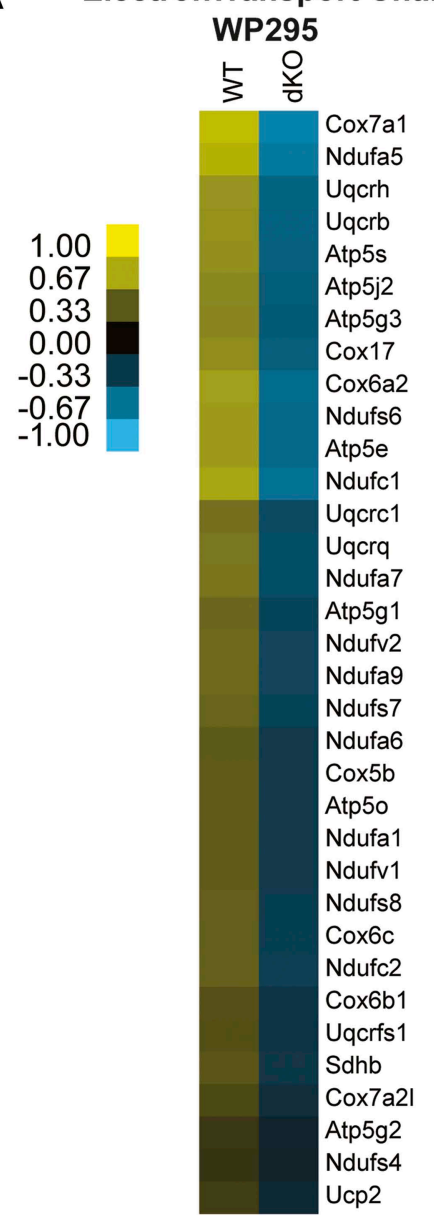

WP1248
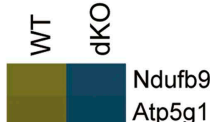

Ndufv2

Ndufa11

Ndufa9

Ndufs 7

Ndufc2

Ndufb8

Ndufb5

Ndufa7

Ndufs2

Atp5o

Ndufv1

Ndufa6

Ndufb6

Atp5g2

Ndufs4

Atp5g3

Atp5j2

Ndufb2

Ndufb7

Ndufs6

Atp5e

Atp5s

Ndufa5

Ndufc1

Ndufc2

Cox6b1

Uqcrfs1

Cox7a2

Atp5g2

Ucp2

\section{Biogenic Amine Synthesis:WP522}

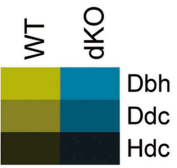

TCA Cycle:WP4347

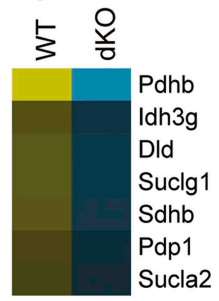

Glycolysis and

Gluconeogenesis:WP157

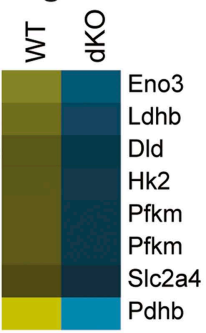

B

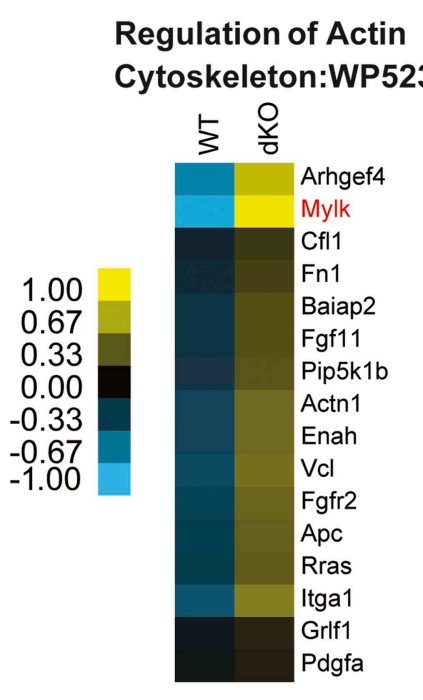

C

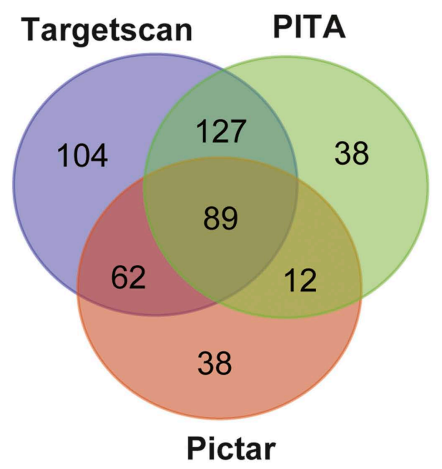

$\log _{2}$

Fold Change

Gene Symbol dKO/WT

\begin{tabular}{|c|c|}
\hline Mylk & 1.7545 \\
\hline Mmd & 1.0988 \\
\hline Edn1 & 0.9883 \\
\hline Gja1 & 0.8574 \\
\hline Hmgn1 & 0.8483 \\
\hline Cpeb1 & 0.8353 \\
\hline Coro1C & 0.7990 \\
\hline Dlg4 & 0.6462 \\
\hline Ppib & 0.5582 \\
\hline Anp32B & 0.5432 \\
\hline Snx2 & 0.4552 \\
\hline Rngtt & 0.4513 \\
\hline Rasa1 & 0.2126 \\
\hline
\end{tabular}

Figure 4. Pathway analysis of genes dysregulated in E18 miR-1 null hearts. (A) Pathway analysis of genes downregulated in E18 miR-1 null hearts identified via Go-elite and GenMAPP. Pathways related to metabolism are enriched. (B) Pathway analysis of genes upregulated in the miR-1 null hearts identified via Go-elite and GenMAPP. Values represent normalized mean centered $\log _{2}$ of FPKM for each genotype. (C) Venn diagram depicting overlap Figure 4. Continued on next page 
Figure 4. Continued

of miR-1 targets as predicted by three prediction algorithms: Targetscan, PITA and Pictar. The genes analyzed were expressed at a relative quantity of $\geq 1$ in miR-1 null vs wild-type hearts based on their FPKM (upper). Consensus targets predicted by all three algorithms, showing significant upregulation in the miR-1 null vs wild-type hearts are presented as descending $\log _{2}$ fold change in miR-1 null over wild-type hearts (lower). Mylk (MLCK) in red was identified as a regulator of the actin cytoskeleton (B) and a significantly upregulated, predicted miR-1 target (C). dKO, double-knockout; WT, wild-type; FPKM, fragments per kilobase per million.

DOI: 10.7554/eLife.01323.026

The following source data and figure supplements are available for figure 4:

Source data 1. miR-1 targets as predicted by three algorithms.

DOI: 10.7554/eLife.01323.027

Figure supplement 1. MicroRNA target enrichment analysis of genes that were expressed at a relative quantity of $\geq 1$ in the miR-1 null vs wild-type hearts. DOI: 10.7554/eLife.01323.028

cell types, a 130-kD broadly expressed isoform and a 17-kD isoform, called Telokin, which lacks a functional kinase domain but shares an identical carboxy-terminal domain, and is specifically expressed in smooth, but not cardiac, muscle. The transcription of each isoform is regulated by individual promoters, which direct their spatially and temporally restricted expression (Herring et alo, 2006). When the $3^{\prime}$ UTR region of Mlck/Telokin containing the predicted miR-1 binding site was cloned downstream of a luciferase reporter, luciferase activity was repressed in the presence of a miR-1 mimic. The repression was alleviated when the target site was deleted, validating this $3^{\prime}$ UTR of Mlck/Telokin as a direct miR-1 target (Figure 5B).

To determine which isoforms were upregulated in miR-1 double-knockout hearts, we examined the protein expression profiles of the various isoforms in wild-type and miR-1 double-knockout hearts by western blot (Figure 5C). The non-muscle 210-kD isoform was undetectable, and the expression of the $130-k D$ MLCK isoform was not significantly altered in miR-1 double-knockout hearts. Telokin protein, as previously reported (Herring et al., 2001), was not detectable in wild-type hearts, but was highly expressed in hearts of miR-1 double-knockouts. qPCR confirmed the normally smooth muscle-restricted Telokin was aberrantly expressed in miR-1 null myocardium (Figure $5 C$ ). Notably, while smooth muscle gene expression is reported to be dysregulated in miR-133a-1:miR-133a-2 double-knockout hearts (Liu et al., 2008), we found that Telokin expression was not upregulated in those animals (Figure 5figure supplement 1), suggesting that the misexpression was specifically due to the loss of miR-1.

Despite lacking a catalytic kinase domain, Telokin plays an important role in the regulation of MLC phosphorylation in smooth muscle by inhibiting MLCK and promoting activity of the MLC phosphatase (Choudhury et al., 2004; Khromov et alo, 2012) (Figure 5D). Consistent with aberrant Telokin expression in cardiomyocytes, MLC phosphorylation was dramatically decreased in miR-1 double-knockout hearts, likely contributing to the observed cardiac dysfunction (Figure 5E).

While direct repression by miR-1 may help to inhibit cardiac translation and stability of Telokin transcripts, it was unclear how loss of miR-1 resulted in the preferential upregulation of Telokin and not the full-length Mlck, which is thought to share a common 3' UTR. To determine if the Telokin promoter was aberrantly active in miR-1 double-knockout hearts, we performed RNA Pol II chromatin immunoprecipitation and assayed for occupancy at the Telokin promoter. In wild-type hearts, Pol II occupancy at the Telokin promoter was equivalent to that of an untranscribed intergenic region, in agreement with Telokin's published smooth muscle-restricted expression pattern (Herring et al., 2001) (Figure 5F). In contrast, Pol II actively bound the Telokin promoter in miR-1 double-knockout hearts (Figure 5F). These data indicate that miR-1 normally acts to repress Telokin expression in the heart by both directly targeting the Telokin 3' UTR and by negatively regulating Telokin transcription.

\section{miR-1 targets the SRF co-factor Myocardin for repression}

To gain mechanistic insight into how miR-1 may be negatively regulating Telokin transcription and to identify, at a more general level, transcriptional networks perturbed in the absence of miR-1, we again utilized the GREAT interface (McLean et alo, 2010) to identify known transcription factor motifs within the Msig database that were enriched within regulatory elements of the genes dysregulated in the double-knockouts. Interestingly, a disproportionate number of the genes upregulated in miR-1 doubleknockout hearts contained CArG boxes (CC/ATn/GG), a motif bound by SRF (Treisman, 1986) (Figure 6A, Figure 6-figure supplement 1). SRF is a critical regulator of the cardiac and smooth muscle transcriptome and regulates sarcomere formation in cardiomyocytes (Li et al., 1997, 2003; Wang et al., 
A
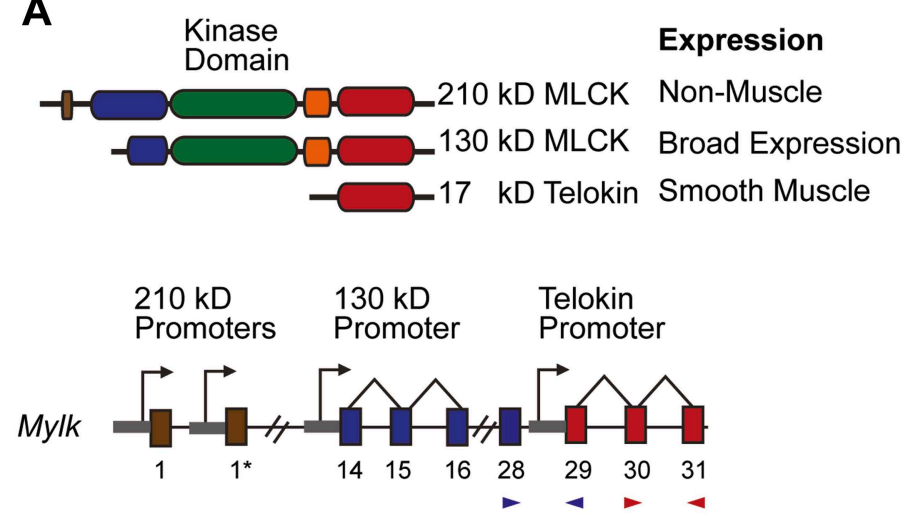

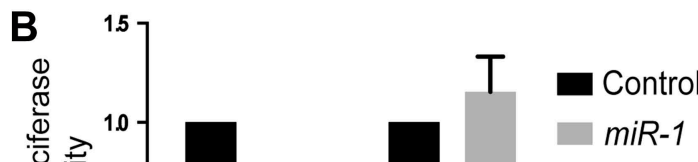

C
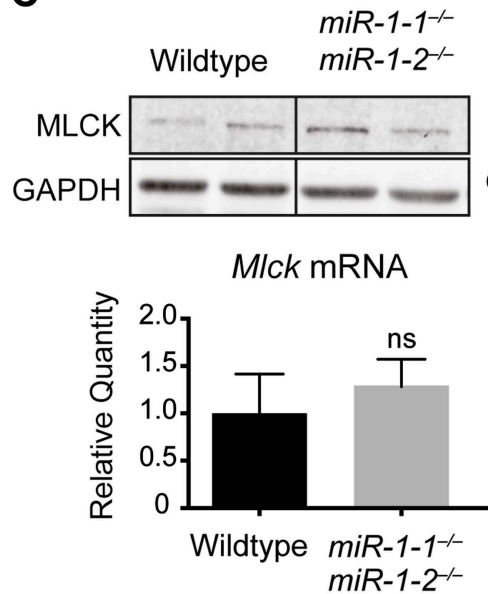

$\operatorname{miR}-1-1^{-1-}$ Wildtype $\operatorname{miR}-1-2^{-1-}$
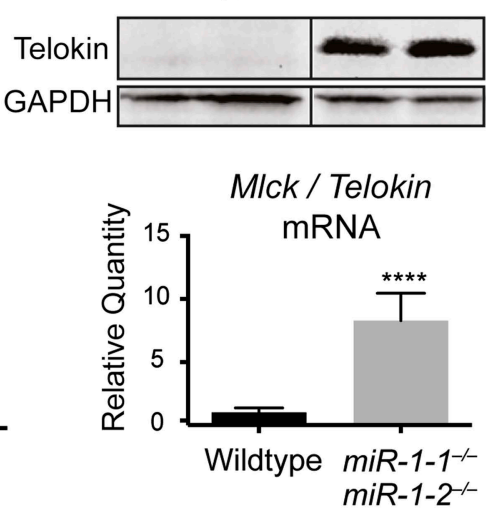

D

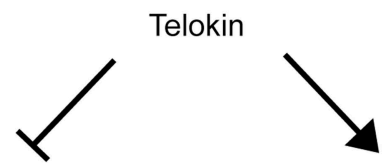

Myosin Light

Chain Kinase

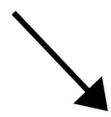

Myosin Light Chain Phosphatase

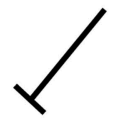

Phospho-Myosin Light Chain
$\mathbf{E}$

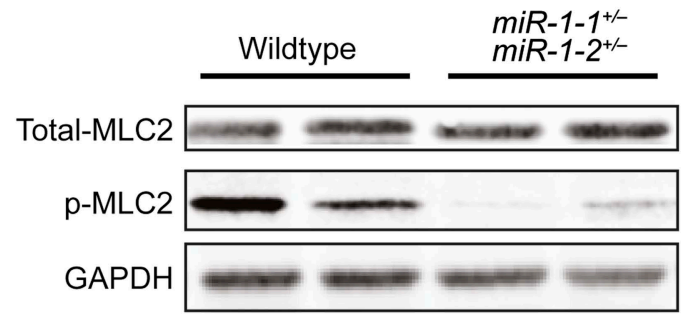

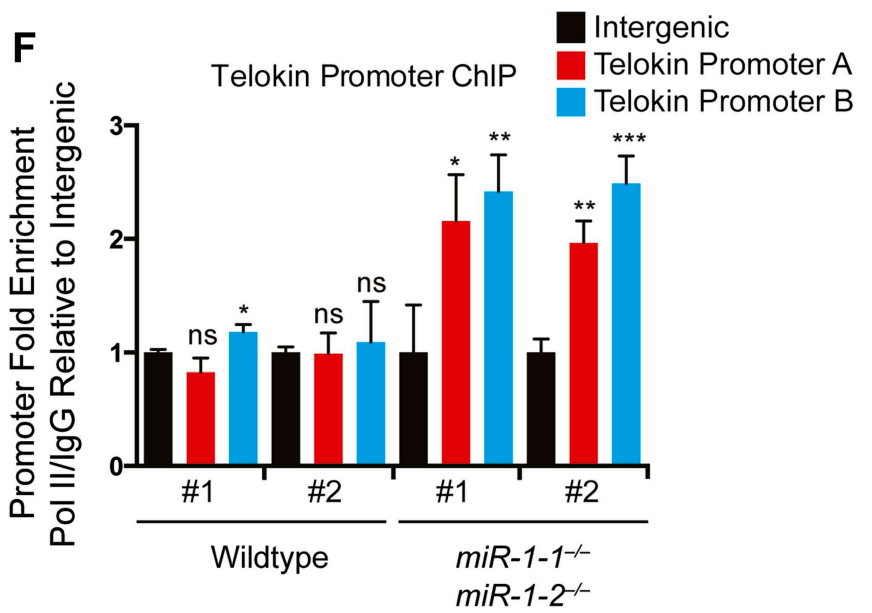

Figure 5. Dysregulation of Telokin and Myosin light chain phosphorylation in miR-1 null hearts. (A) Diagram of the gene products encoded within the Mylk locus. Independent promoters preceding exons are indicated by arrows. Exon-spanning qPCR primers used are indicated in red (Mlck/Telokin) or blue (Mlck). (B) Luciferase activity of a reporter construct containing 200 bp of the Mlck/Telokin 3'-UTR surrounding the predicted miR-1 binding site Figure 5. Continued on next page 
Figure 5. Continued

with or without the site deleted. The constructs were co-transfected into H9C2 myoblasts with a miR-1 mimic or a control mimic. Sequence of the putative miR-1 target site as predicted by Targetscan and site conservation between human ( $\mathrm{Hs}$ ) and mouse (Mm) is indicated. (C) Western blot of heart lysates (top) and qPCR of RNA (bottom) from P0 wild-type or miR-1 null mice. ( $N=5$ per group). (D) Model of Telokin function in smooth muscle to promote the activity of myosin light chain phosphatase and inhibit the activity of the myosin light chain kinase. (E) Western blot of total myosin light chain 2 (MLC2) and phosphorylated myosin light chain ( $p$-MLC2) in P0 wild-type or miR-1 null hearts; GAPDH serves as loading control. (F) qPCR of the Telokin promoter sequence or an intergenic genomic sequence following chromatin immunoprecipitation (ChIP) of RNA polymerase II in P2 wild-type or miR-1 null hearts. For the Telokin promoter, two non-overlapping probe sets were used, indicated as Telokin promoter A and B.

DOI: 10.7554/eLife.01323.029

The following figure supplements are available for figure 5:

Figure supplement 1. Putative miR-1 targets dysregulated in miR-1 null hearts were not affected in miR-133a double-knockout hearts. DOI: 10.7554/eLife.01323.030

2001; Niu et al., 2007; Hoofnagle et al., 2011). SRF is also a highly conserved direct upstream regulator of the miR-1/133a transcript (Kwon et al., 2005; Zhao et al., 2005) and is itself a target of miR-133a, being upregulated in the hearts of miR-133a-1:miR-133a-2 double-knockout animals (Chen et al., 2005; Liu et al., 2008). However, SRF was not dysregulated at the RNA or protein level in miR-1 doubleknockout hearts (Figure 6B). This finding suggests that a simple increase in SRF expression is not responsible for the upregulation of SRF target genes and, furthermore, that the level of miR-133a expressed in miR-1 double-knockouts is sufficient to maintain normal SRF expression levels.

One mechanism by which the specificity of SRF targets is conferred is through the expression and activity of tissue-specific cofactors. Myocardin (MYOCD), which is expressed predominantly in cardiac and smooth muscle (Wang et al., 2001), is one such cofactor. MYOCD is necessary and sufficient for smooth muscle differentiation in vitro and in vivo (Li et al., 2003; Chen et al., 2002; Wang et al., 2003) and is necessary for the differentiation and maintenance of ventricular cardiomyocytes (Wang et al., 2001; Hoofnagle et al., 2011). Among the SRF target genes that were dysregulated in the miR-1 double-knockouts, many, including Telokin, were known targets of the MYOCD/SRF complex (Figure 6C, Figure 6-figure supplement 1), while MYOCD independent SRF target genes, such as Fos and Egr1, were not upregulated (Figure 6C). Notably, the expression of numerous other known SRF co-factors, including NKX2-5, MRTF-A, BRG1, GATA4 and GATA6 were unchanged (Figure 6-figure supplement 2). These findings suggest that MYOCD/SRF dependent gene expression is specifically upregulated in the miR-1 knockout hearts.

Using qPCR to compare the expression of Myocd in miR-1 double-knockout hearts and controls, we found that the expression of this critical transcription factor was increased roughly twofold upon deletion of miR-1 (Figure 7A). Using Targetscan, we identified a potential miR-1 binding site in the Myocd 3'-UTR and demonstrated, using a luciferase assay, that miR-1 directly targets the Myocd 3' UTR (Figure 7B). Previous studies have shown that the Telokin promoter is more responsive to MYOCD than the full-length 130-kD Mlck promoter (Herring et al., 2006). Thus, as we observed, an increase in MYOCD expression would be predicted to preferentially upregulate the transcription of the Telokin isoform, while only modestly increasing transcription of the full-length transcript.

\section{Smooth muscle Myocd expression is upregulated in miR-1 double- knockout hearts}

There are two distinct Myocd isoforms, the full-length cardiac isoform (cMYOCD) and the truncated smooth muscle isoform (smMYOCD) (Creemers et al., 2006). smMyocd is produced by alternate splicing and inclusion of exon2a, which encodes a translational stop and necessitates the use of an alternate downstream start site. This splicing event results in a truncated protein, lacking the N-terminal-MEF2 interaction domain of the full-length CMYOCD. Although the functional significance of these two isoforms is not well understood, the promoters of some smooth muscle-restricted genes, such as Tagln (Sm22), are more sensitive to smMYOCD than CMYOCD, while some cardiac promoters, such as Myh6 ( $\alpha-M h c)$, are more responsive to CMYOCD (Imamura et al., 2010). Normally, smMyocd expression is relatively low in the neonatal heart; however, we discovered using qPCR that in miR-1 double-knockout hearts the expression of smMyocd was fivefold higher than control hearts, while cMyocd was upregulated roughly 1.8-fold (Figure 7A). More specific upregulation of smMyocd was not evident with statistical confidence in our RNA-seq data likely due to insufficient sequencing depth to detect the small $44 \mathrm{bp}$ exon $2 a$ inclusion within the relatively low overall abundance of smMyocd transcript. 
A

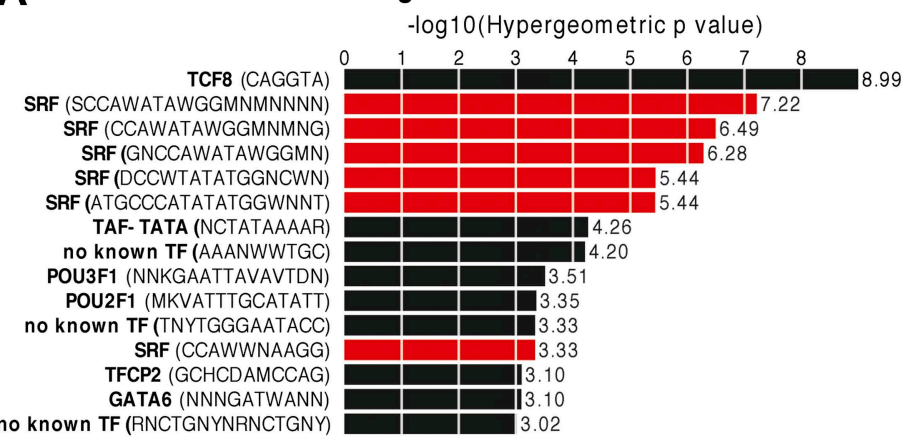

C

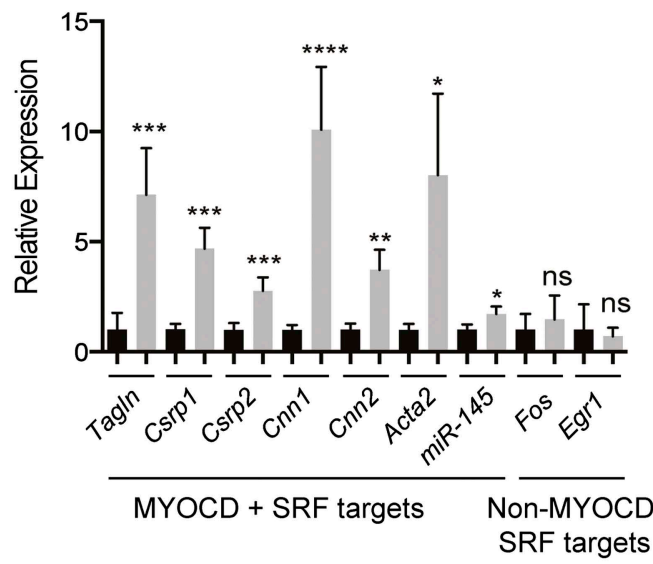

B

$\operatorname{miR}-1-1^{-1}$

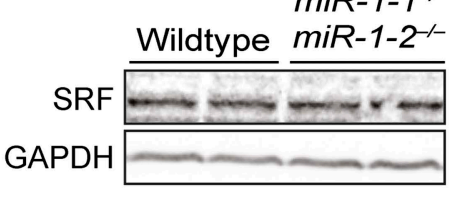

Srf mRNA

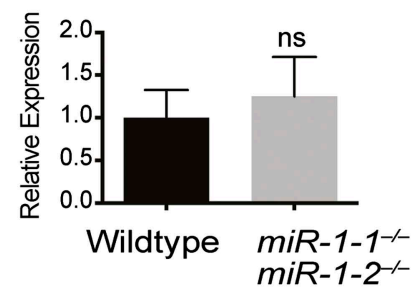

miR-1-1-1Wildtype $\operatorname{miR}-1-2^{-1-}$

Wildtype $\quad \operatorname{miR}-1-1^{-/}: \operatorname{miR}-1-2^{-/}$

Figure 6. Upregulation of SRF targets in miR-1 null hearts. (A) Promoter motif enrichment in genes upregulated in miR-1 double-knockout hearts. Motif sequences and transcriptional regulators are indicated (left) with the $\log _{10}$ of the hypergeometric $p$ value graphed. Multiple CArG box sequences, the motif bound by SRF, were identified (red). (B) Western blot (top), and qPCR (bottom) for Srf expression in post-natal miR-1 null or wild-type hearts. (C) Myocardin dependent and independent SRF target gene expression in postnatal miR-1 null or wild-type hearts by qPCR (left). Western blots of selected Myocardin-dependent SRF target genes (right). Tagln, Transgelin/Sm22; Csrp1,2, Cysteine And Glycine-Rich Protein 1,2; Cnn1,2, Calponin1,2; Acta2, smooth muscle alpha actin; miR-145, microRNA-145; Fos, FBJ Murine Osteosarcoma Viral Oncogene Homolog; Egr1, Early growth response 1. ${ }^{*} \mathrm{p}<0.05 ;{ }^{* \star} \mathrm{p}<0.01 ;{ }^{* \star *} \mathrm{p}<0.001 ;{ }^{* \star * *} \mathrm{p}<0.0001 ;$ ns, not significant.

DOI: 10.7554/eLife.01323.031

The following figure supplements are available for figure 6:

Figure supplement 1. Dysregulation of SRF targets in miR-1 double-knockout hearts.

DOI: 10.7554/eLife.01323.032

Figure supplement 2. (A) qPCR of SRF co-factors in miR-1 wild-type or double-knockout hearts. $N=5$. (B) Western blot for protein expression of SRF co-factors. ns, not significant. DOI: 10.7554/eLife.01323.033

While cMyocd still represents the predominant isoform in miR-1 double-knockout hearts (data not shown), subtle changes in transcription factor expression can result in dramatic changes in gene expression. Using a luciferase reporter cloned downstream of the mouse Telokin promoter, we found that smMYOCD was twice as effective as CMYOCD at activating the Telokin promoter (Figure 7C). More broadly, we found that many of the MYOCD-dependent genes upregulated in the miR-1 knockouts were smooth muscle genes (Figure 6-figure supplement 1). Smooth muscle gene upregulation was also reported in the myocardium of miR-133a knockout mice, due in part to the upregulation of SRF. Neither total Myocd nor smMyocd, however, was upregulated in the myocardium of miR-133a doubleknockouts, indicating that the miR-133a levels do not affect Myocd expression (Figure 5-figure supplement 1). These data suggest a model whereby miR-1 and miR-133a cooperate to repress 


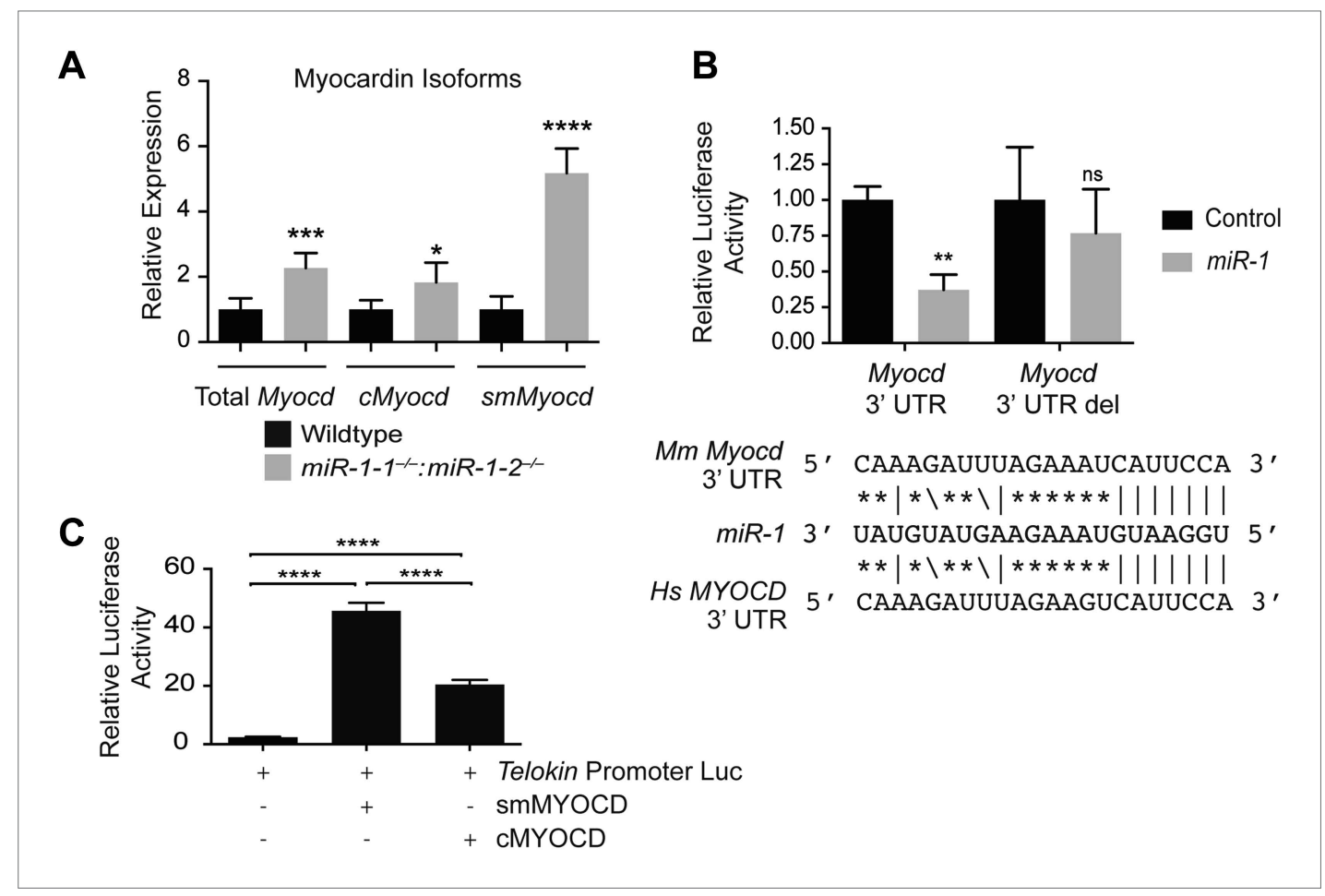

Figure 7. miR-1 regulates the SRF co-factor, Myocardin. (A) qPCR for Myocardin (Myocd) expression. Probe sets are specific for inclusion of exon 2a for smooth muscle Myocd (smMyocd), exclusion of exon 2a for cardiac Myocd (cMyocd), or for downstream exons common to both transcripts (Total Myocd). PO hearts were analyzed, $\mathrm{N}=5$ per genotype. (B) Luciferase activity of a reporter construct containing the miR-1 putative target site derived from the Myocd $3^{\prime}$ UTR or a deleted site. The constructs were co-transfected into H9C2 myoblasts with either a miR-1 mimic or a control mimic. The sequence of the putative miR-1 target site as predicted by Targetscan and site conservation between human $(\mathrm{Hs})$ and mouse $(\mathrm{Mm})$ is indicated. (C) The expression of luciferase driven by the Telokin +370 promoter when transfected into Cos cells, alone or with either full-length smMyocd or cMyocd. ${ }^{*} p<0.05 ;{ }^{* \star} p<0.01$; ${ }^{* * *} p<0.001 ; * \star * \star p<0.0001 ;$ ns, not significant. DOI: 10.7554/eLife.01323.034

smooth muscle gene transcription in the heart by repressing smMyocd and Srf, respectively, thereby reinforcing the striated muscle phenotype (Figure $8 \mathrm{~B}$ ).

\section{Discussion}

The results of this study reveal the essential, multi-faceted role that miR-1 plays in the mammalian heart. The uniform lethality of miR-1 double-knockout animals, associated with dilated cardiomyopathy and abnormal heart rhythm, indicates that miR-1 is required for normal cardiac contractility. At a cellular level, we found that sarcomere disruption is one feature that underlies the impaired cardiac function, and reintroduction of miR-1 into ex vivo cardiomyocytes was sufficient to partially rescue this phenotype. miR-1 functions to negatively regulate the smooth muscle-specific inhibitor of MLC phosphorylation, Telokin. Aberrant upregulation of Telokin and the resulting decrease in phosphorylated MLC in miR-1 null hearts may contribute, in part, to the sarcomeric contractility defect. miR-1 regulation of Telokin is accomplished by direct targeting of its $3^{\prime}$ UTR, as well as through targeting of its transcriptional regulator, Myocd. In particular, miR-1 preferentially regulates smMyocd, thereby complementing the effects of miR-133 to suppress the smooth muscle gene program (Figure 8).

\section{miR-1 is required for normal sarcomere formation and maintenance}

In ex vivo cardiomyocytes, restoring miR-1 expression alone was sufficient to partially rescue the sarcomeric defects of miR-1 double-knockout hearts, demonstrating that the loss of miR-1 plays a causative role in this phenotype, but not ruling out a contribution from decreased miR-133a expression. In this and previous studies (Mishima et al., 2009), genes related to the actin cytoskeleton are highly responsive to alterations in miR-1 expression. MLC phosphorylation is critical for sarcomere assembly 


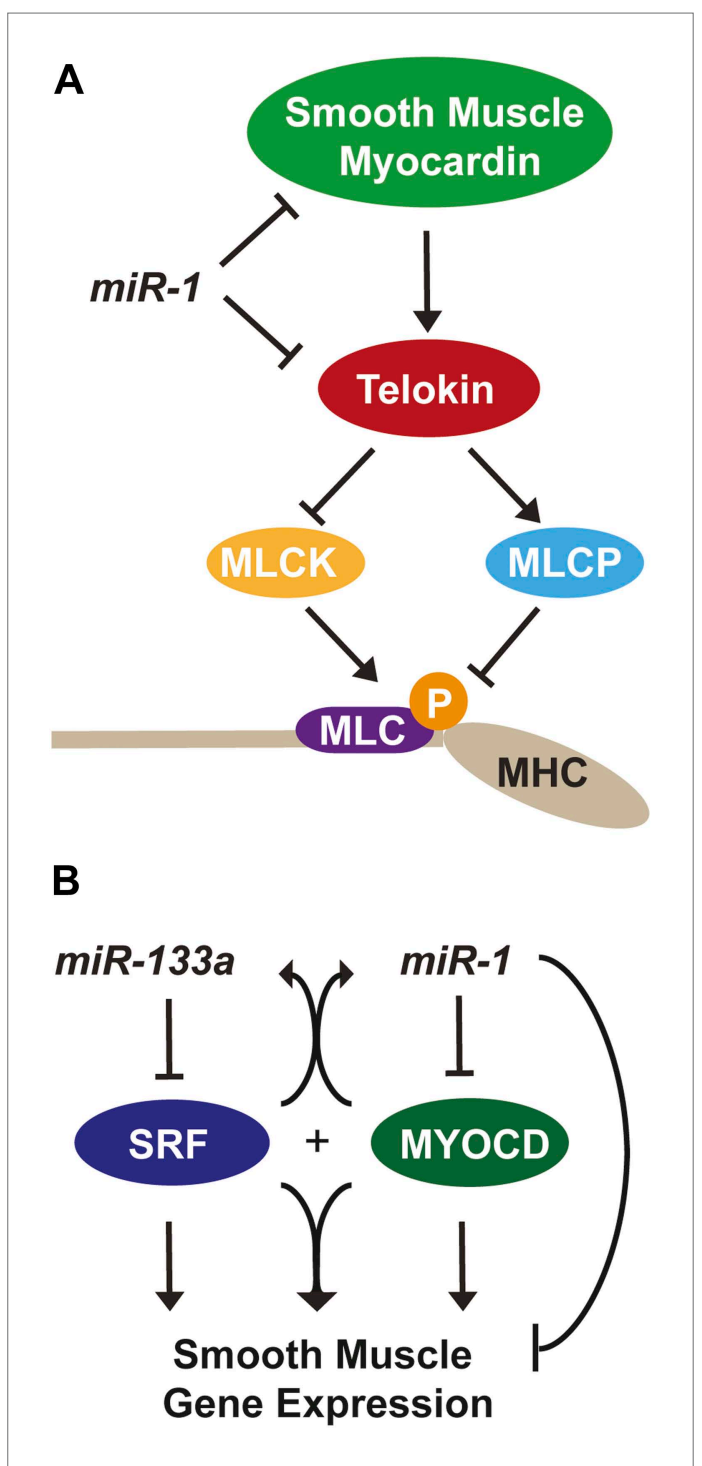

Figure 8. miR-1 regulation of Myosin light chain phosphorylation and smooth muscle gene expression. (A) Dual regulatory model by which miR-1 acts to normally repress Telokin expression in the heart by directly targeting its $3^{\prime}$ UTR and its upstream transcriptional regulator, smooth muscle myocarding (smMYOCD). MLCK, myosin light chain kinase; MLCP, myosin light chain phosphatase; MLC, myosin light chain; MHC, myosin heavy chain; P, phosphorylation. (B) Regulatory model by which the miR-1/133a cluster cooperates to suppress smooth muscle gene expression in the heart. DOI: 10.7554/eLife.01323.035 understanding of the role that the miR-1 family plays in the context of skeletal muscle will require the generation a skeletal muscle-specific compound deletion of miR-1-1, miR-1-2 and miR-206.

\section{miR-133a downregulation in miR-1 null hearts}

The marked downregulation of miR-133a in the miR-1 double-knockouts is a critical consideration with respect to the interpretation of some of the phenotypes that we observed. While targeted disruption
of miR-1 might adversely affect transcriptional read-through of the $m i R-1-1 / m i R-133 a-2$ locus, respect to the interpretation of some of the phenotypes that we observed. While targeted disruption
of miR-1 might adversely affect transcriptional read-through of the miR-1-1/miR-133a-2 locus,

and regulating the speed and force of contraction in the heart (Aoki et al., 2000; Davis et al., 2001). In mammals, there are multiple MLC2 isoforms, but deletion of the single cardiac zebrafish isoform results in a lack of thick filament assembly (Rottbauer et al., 2006). At a molecular level, phosphorylation of MLC2 results in a conformational shift in the associated $\mathrm{MHC}$, bringing it into closer proximity to the thin filament and increasing the probability of cross-bridge formation. Not surprisingly, in humans, MLC2 mutations are associated with myopathies (Poetter et al., 1996; Davis et al., 2001). Thus, decreased phosphorylation of MLC in the absence of miR-1 likely plays a role in the sarcomeric disruption and cardiac dysfunction in miR-1 double-knockouts.

We found that one mechanism by which miR-1 normally acts to maintain P-MLC in the heart is through the repression of the usually smooth muscle-restricted protein Telokin. Telokin expression in smooth muscle is thought to maintain cells in a less contractile state, and it is interesting that the heart employs miR-1 to ensure the absence of Telokin through both transcriptional and posttranscriptional mechanisms (Figure 8A). Although it is not possible to distinguish the contribution of transcriptional regulation of Telokin vs direct miR-1 targeting of the Telokin 3' UTR, the dramatic degree of Telokin protein upregulation compared to the more moderate Telokin mRNA increase is consistent with some degree of translational control by miR-1 (Figure 4C). How Telokin misexpression affects cardiac function has not been directly evaluated; however, ectopic expression in the myocardium has been reported in a doxorubicin induced rat model of cardiomyopathy, although the precise mechanism by which Telokin contributes to this pathology is unknown (Dudnakova et al., 2003).

While the focus of this study was to explore the function of miR-1 in the heart, miR-1 likely plays a more subtle role in the skeletal muscle. It is worth noting that electron microscopy of PO miR-1-null skeletal muscle showed the presence of ordered sarcomeric structures (data not shown) and neonatal mice were mobile. While we cannot rule out a more subtle skeletal muscle phenotype due to the early lethality, it is possible that the skeletal muscle specific miR-1 family member, miR-206, can compensate for the loss of miR-1 in this tissue. A clear 
secondary downregulation of miR-133a expression from regulatory feedback mechanisms also appears to occur (Figure 2-figure supplement 10). miR-1 and miR-133a are dramatically upregulated during the differentiation of cardiac and skeletal muscle (Chen et al., 2005; Ivey et al., 2008). While the current study shows definitively that miR-1 is not required for initial cardiomyocyte differentiation in vivo, the loss of miR-1 expression may impair further cardiomyocyte differentiation and/or maturation. This would predictably result in decreased developmental upregulation of the miR-1/133 loci. Consistent with this model, the loosely organized sarcomeric morphology and the abnormal mitochondrial morphology we observed by TEM are similar to those of more immature cardiomyocytes (Hom et al., 2011). The upregulation of members of the cardiac fetal gene expression program, such as Nppa (Figure 6-figure supplement 1), and even the upregulation of smooth muscle genes, which are normally expressed in embryonic cardiomyocytes, supports this paradigm. As such, the expression of miR-133a, a key developmentally regulated miRNA, may be secondarily downregulated in miR-1 doubleknockout mice as a result of a subtle cardiac differentiation or maturation defect, although the exact mechanism is unknown.

\section{The miR-1 and miR-133a clusters cooperate to normally repress smooth muscle gene expression in the heart}

The power of miRNAs to affect biological processes is amplified by the ability of a single miRNA to regulate multiple nodes within a genetic network. Srf is a direct target of miR-133a, and its expression, as well as that of many smooth muscle target genes, is upregulated in the hearts of miR-133a-knockout animals (Chen et alo, 2005; Liu et alo, 2008). We found that SRF mRNA and protein expression in miR-1 double-knockout hearts was unaffected, despite the partial downregulation of miR-133a. Nevertheless, some of the smooth muscle genes upregulated in miR-133a double-knockout hearts were also upregulated in the miR-1 double-knockout animals (Acta2, Cald1, Csrp2, Cnn1, Tagln), while others, including Telokin, cMyocd and smMyocd, were uniquely upregulated in the miR-1 double-knockouts. Our data suggest that miR-1 and miR-133a, transcribed together from bicistronic miRNA clusters, cooperate to repress SRF-dependent smooth muscle gene expression in the heart by independently regulating SRF or its co-factor, MYOCD, respectively (Figure 8B).

We also found that the Telokin promoter is preferentially responsive to the smMYOCD isoform, upregulated in the absence of miR-1. This is consistent with the observation that, while both MYOCD isoforms share an identical SRF-interacting domain, they are not functionally equivalent with respect to mediating the transcription of SRF target genes (Creemers et al., 2006; Imamura et al., 2010). While yet to be carefully evaluated, cMyocd and smMyocd are thought to share a common 3' UTR; thus, it is unlikely that direct targeting by miR-1 alone is responsible for the observed isoform switch. The splice factor(s) that regulate the inclusion or exclusion of the $2 a$ exon in the smooth muscle or heart, respectively, are unknown. Therefore, a potential mechanism by which miR-1 may regulate Myocd splicing is through the direct repression of a splice factor that normally mediates alternate inclusion of the 2a exon in smooth muscle. Further studies will be required to elucidate the mechanism by which miR-1 regulates this critical transcription factor and ultimately smooth muscle gene expression.

\section{Materials and methods}

\section{miR-1-1 gene targeting and mouse breeding}

The miR-1-1 targeting vector was generated by flanking $5^{\prime}$ and $3^{\prime}$ genomic DNA around a floxed neomycin resistance gene (Neo) driven by a pGK promoter. Targeting of the miR-1-1 locus was accomplished through homologous recombination in E14 ES cells and resulted in replacement of the genomic sequence containing the pre-miR-1-1 ( 280 bp) by the Neo gene. 672 colonies were screened, and three targeted clones were identified. miR-1-1 heterozygous embryonic stem cells were injected into D3.5 BL6 blastocysts. For the establishment of the miR-1-1-targeted allele on a 129 background, chimeric males were mated to wild-type 129 (Jackson Lab) females. To generate compound miR-1 knockouts, miR-1-1-targeted animals were crossed to a previously described miR-1-2 targeted mouse maintained on a mixed (129/BL6) background. Animals double heterozygous for both miR-1 alleles $\left(m i R-1-1^{+/}: m i R-1-2^{+/-}\right)$were subsequently intercrossed to generate animals lacking three out of four

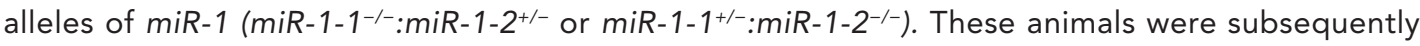
intercrossed to generate compound miR-1 knockout animals (miR-1-1-1-- $\left.m i R-1-2^{--}\right)$. Genotyping was 
performed by PCR [Supplementary file 1] with primers that specifically recognized the wild-type or targeted miR-1-1 or miR-1-2 alleles.

The animals were sacrificed by decapitation for neonates or by $\mathrm{CO}_{2}$, followed by cervical dislocation for adult animals. All animal care and experimental protocols were reviewed and approved by the Institutional Animal Care and Use Committee of the University of California, San Francisco (UCSF).

\section{Electron microscopy}

For electron microscopy, tissue was fixed in $2 \%$ glutaraldehyde, $1 \%$ paraformaldehyde in $0.1 \mathrm{M}$ sodium cacodylate buffer $\mathrm{pH} 7.4$, post fixed in $2 \%$ osmium tetroxide in the same buffer, en block stained in $2 \%$ aqueous uranyl acetate, dehydrated in acetone, infiltrated, and embedded in LX-112 resin (Ladd Research Industries, Williston, VT). Toluidine blue stained semi-thin sections were made to locate the areas of interest. The samples were ultrathin sectioned on a Reichert Ultracut $\mathrm{S}$ ultramicrotome and counter stained with $0.8 \%$ lead citrate. Grids were examined on a JEOL JEM-1230 transmission electron microscope (JEOL USA, Inc., Pleasanton, CA) and photographed with the Gatan Ultrascan 1000 digital camera (Gatan Inc., Pleasanton, CA).

\section{Neonatal transthoracic echocardiography and electrocardiogram}

Adult mouse echocardiography was performed under anesthesia as described (Qian et al., 2011). Electrocardiograms were performed as described (Zhao et al., 2007). Neonatal studies were performed as described above without anesthesia.

\section{Histology and immunocytochemistry}

Tissue was collected at indicated times and fixed using $10 \%$ formalin overnight at $4{ }^{\circ} \mathrm{C}$ and stored subsequently in $70 \%$ ethanol. Paraffin embedding and staining was performed using standard histological techniques. Sarcomeric staining was performed using anti-sarcomeric alpha-actinin (1:400; Sigma, St. Louis, MO) and rhodamine conjugated Phalloidin (Clontech, Mountain View, CA).

\section{Isolation and culture of primary neonatal cardiomyocytes}

Hearts were isolated from P0 animals and rinsed several times in 1X HBSS with Pen-Strep. The great vessels and atrium were removed and discarded, and the ventricles were minced manually with scissors and further disassociated enzymatically with collagenase digestion. $1 \mathrm{mg} / \mathrm{ml}$ collagenase II was added to the minced ventricles and briefly incubated at $37^{\circ} \mathrm{C}$ for $3-5 \mathrm{~min}$ with agitation. The digests were allowed to settle without agitation briefly $(3 \mathrm{~min})$ before the supernatant of this initial digestion (mostly blood cells) was discarded and another digestion was performed for 15-20 min. The supernatant of this subsequent digestion (cardiomyocytes) was added to 3X volume of FBS, and centrifuged for 5 min at $300 \times g$ in a tabletop centrifuge. The resulting cell pellet was resuspended in DMEM/F12 medium with $10 \%$ FBS and Pen-Strep and passed through a $70-\mu \mathrm{M}$ filter before plating on $1 \%$ gelatin with fibronectin. Cardiomyocytes for sarcomeric analysis were fixed and stained $24 \mathrm{hr}$ post-plating. The cardiomyocytes used for sarcomeric rescue studies were transfected with miR-1 mimic or control (Ambion/Life Technologies, Carlsbad, CA) roughly $12 \mathrm{hr}$ post-plating with Lipofectamine 2000 (Life Technologies) and maintained in serum-containing medium. The cells were fixed for analysis $24 \mathrm{hr}$ post-transfection.

\section{Cloning, plasmids, transfection and luciferase assays}

Roughly 200 bp surrounding the predicted miR-1 target sites in the Mlck/Telokin or Myocd 3' UTR were amplified directly from cDNA generated from miR-1 double-knockout hearts with the primers listed (Supplementary file 1) and subcloned into the PGL3 (Promega, Madison, WI) firefly luciferase vector $3^{\prime}$ of the reporter gene with Xbal.

Luciferase constructs were transfected along with a Renilla normalization vector into the H9C2 rat myoblast cell line with Lipofectamine 2000 (Life Technologies), according to manufacturer's instructions. Briefly, 12-well plates were transfected at roughly $60 \%$ confluency and analyzed $20 \mathrm{hr}$ later. Each well received $3 \mu$ l of Lipofectamine 2000, $800 \mathrm{ng}$ of PGL3-Target, and $200 \mathrm{ng}$ of Renilla vector. Experimental wells received 10 pmols of miR-1 mimic (Ambion/Life Technologies), while control wells received 10 pmols of a non-targeting control mimic (Ambion/Life Technologies). Promoter activity assays were performed with the Telokin+370 promoter (a gift from Dr Paul Herring, Indiana University). cMyocd and smMyocd constructs were as described in Wang and Olson (2004) Cordes et al., (2009). Promoter constructs (500 ng), Myocd (250 ng) or control LacZ expression plasmid and 50 ng of Renilla were transfected into Cos cells with Lipofectamine 2000 at $50 \%$ confluency. Luciferase intensity was analyzed $20 \mathrm{hr}$ post-transfection. 
Firefly and Renilla luciferase activities were quantified in lysates with the Dual Luciferase Reporter Assay kit (Promega) on a Victor 1420 Multilabel Counter (PerkinElmer, Madison, WI). Firefly luciferase values were normalized to Renilla to control for transfection efficiency.

\section{Quantitative real-time PCR}

RNA was isolated with TRIzol reagent according to the manufacturer's protocol. Quantitative real-time PCR for microRNAs was performed with the TaqMan miRNA assay kit (Applied Biosystems/Life), and TaqMan probes for miR-1 (Applied Biosystems/LIfe), miR-133a (Applied Biosystems/Life) were used according to the manufacturer's protocols. cDNA for mRNA quantification was generated using Superscript with oligodT and random hexamers (Invitrogen). Detection of splice variants for Myocd was performed using oligo dT generated cDNA (Invitrogen/Life). qPCR probes for the Telokin promoter, and Myocd splice variants were designed using Primerquest software and synthesized by Integrated DNA Technology (IDT, San Jose, CA). Intergenic region primers were designed based on sequence published previously. The probe sequences and part numbers are listed in Supplementary file 1. Expression values were normalized to the expression of U6 (Applied Biosystems/Life) for miRNA analysis or Gapdh (Applied Biosystems/Life) for mRNA quantification, and fold change was determined using the $\triangle \triangle C T$ method with SDS RQ Manager software (Applied Biosystems/Life).

\section{RNA sequencing}

Whole hearts from E18 wild-type and miR-1 double-knockout animals were isolated and total RNA was extracted with TRIzol (Invitrogen/Life), following the manufacturer's suggested protocol. Genomic DNA was removed using a gDNA eliminator column (Qiagen, Hilden, Germany). RNA from three hearts of each genotype was pooled, and library preparation and sequencing were performed by the Beijing Genomics Institute (BGI). In brief, polyA transcripts were enriched and paired-end reads were sequenced on a Highseq 2000 (Illumina, San Diego, CA). Reads were mapped to the mm 9 genome, Ensembl v 59 annotation, with TopHat2 (Kim et al., 2013). Rank expectation (Thomas et al., 2011) was used to identify genes that were differentially expressed between the two backgrounds, using a false discovery rate threshold of 0.1.

\section{Array methods}

Neonatal hearts (P2) from wildtype, miR-1-1 null and miR-1-2+/-:miR-1-1+1- double heterozygote animals were isolated and total RNA was extracted with TRIzol (Invitrogen/Life), following the manufacturer's suggested protocol. Genomic DNA was removed using a gDNA Eliminator column (Qiagen). The samples were hybridized to Affymetrix Mouse Genechip ST 2.0 arrays. All arrays were RMA normalized and differentially expressed genes were identified using Limma. Gene changes were compared to an existing data set (Zhao et al., 2007) for miR-1-2 null animals. 822 genes were statistically changed in one of the three genotypes when compared to the wild-type control ( $p$ value of 0.0025 and a fold change of greater than 1.5 or less than 0.5 ). Of these differentially expressed genes, 201 genes could be evaluated across all genotypes due to the difference in array probe sets.

\section{Western blots}

Hearts were isolated from animals of the indicated genotypes between P0 and P3 and rinsed with 1 X PBS. The tissue was resuspended in RIPA buffer and disassociated in a Bullet Blender (Next Advance). After a clarification and sonication step, the lysates were loaded on to a 4-20\% SDS-PAGE (Biorad, Hercules, CA) gel and blotted using standard protocols. Primary antibodies against GAPDH (1:1000; Abcam), SRF (1:200; Santa Cruz Bio., Dallas, TX), CSRP1 (1:500; Abcam), TAGLN/Sm22 (1:500; Abcam), p-MLC ser18/thr19 (1:1000; Cell Signaling), MLCK/Telokin (1:1000; Abcam, Cambridge, England), ACTA2 (1:2000; Sigma). Visualization and quantification of blots was done on a Licor Odyssey system with fluorescently conjugated secondary antibodies (Licor, Lincoln, NE), according to manufacturer's instructions.

\section{Polymerase II chromatin immunoprecipation}

Pol II ChIP was performed as in Lee et al. (2006) with minor modifications. QPCR primer sequences described in Supplementary file 1.

\section{Bioinformatics and statistical analysis}

Data were analyzed with Prism and/or Excel and an unpaired $t$ test was used to determine statistical significance. Predicted genotype ratios were calculated by chi-square analysis. Relative quantity for qPCR analysis was determined using $\triangle \triangle C T$ method. Values for experimental animals normalized to the average of controls. 


\section{Acknowledgements}

The authors thank G Howard and AL Lucido for their editorial comments; B Taylor for help with manuscript and figure preparation; K Cordes Metzler, I King, V Vedantham and M White for critical discussion; A Williams, S Thomas, and A Holloway of the Gladstone Bioinformatics Core, J Wong of the Gladstone Electron Micropscopy Core, and C Miller and K Bummer of the Gladstone Histology Core for technical support. The Telokin promoter construct was a gift from P Herring. RNA from miR-133a knockout animals was kindly provided by N Liu and EN Olson. The Gladstone Institutes received support from a National Center for Research Resources Grant RR18928.

\section{Additional information}

Competing interests

DS: Member of the Scientific Advisory Boards of iPierian and RegeneRx Biopharmaceuticals, and Reviewing editor, eLife. The other authors declare that no competing interests exist.

Funding

\begin{tabular}{lll} 
Funder & $\begin{array}{l}\text { Grant reference } \\
\text { number }\end{array}$ & Author \\
\hline National Science Foundation & $\begin{array}{l}\text { U01 HL100406, } \\
\text { U01 HL098179, } \\
\text { R01 HL057181, } \\
\text { P01 HL089707 }\end{array}$ & Amy Heidersbach \\
\hline National Institutes of Health & & \\
\hline American Heart Association & & Yen-Sin Ang, \\
& & Kathryn N Ivey \\
\hline Amyotrophic Lateral Sclerosis & Kathryn N Ivey \\
\hline Association & Deepak Srivastava \\
\hline $\begin{array}{l}\text { California Institute for } \\
\text { Regenerative Medicine }\end{array}$ & Deepak Srivastava \\
\hline $\begin{array}{l}\text { William Younger Family } \\
\text { Foundation }\end{array}$ & Deepak Srivastava \\
\hline LK Whittier Foundation & Deepak Srivastava \\
\hline Roddenberry Foundation &
\end{tabular}

The funders had no role in study design, data collection and interpretation, or the decision to submit the work for publication.

Author contributions

$\mathrm{AH}, \mathrm{KNI}$, Conception and design, Acquisition of data, Analysis and interpretation of data, Drafting or revising the article; CS, KC-M, YH, Acquisition of data, Drafting or revising the article; Y-SA, Analysis and interpretation of data, Drafting or revising the article; DS, Conception and design, Analysis and interpretation of data, Drafting or revising the article; PDJ, Made the miR-1-1 targeting vector used in this study

Ethics

Animal experimentation: This study was performed in strict accordance with the recommendations in the Guide for the Care and Use of Laboratory Animals of the National Institutes of Health. All of the animals were handled according to approved institutional animal care and use committee (IACUC) protocols (\#AN086606) of the University of California, San Francisco.

\section{Additional files}

Supplementary files

- Supplementary file 1. Probe sequence/part number.

DOI: 10.7554/eLife.01323.036 
Major dataset

The following datasets were generated:

\begin{tabular}{|c|c|c|c|c|}
\hline Author(s) & Year & Dataset title & Dataset ID and/or URL & $\begin{array}{l}\text { Database, license, } \\
\text { and accessibility } \\
\text { information }\end{array}$ \\
\hline $\begin{array}{l}\text { Heidersbach A, Saxby C, } \\
\text { Carver-Moore K, Huang Y, } \\
\text { Ang Y-S, Ivey KN, } \\
\text { Srivastava D }\end{array}$ & 2013 & $\begin{array}{l}\text { microRNA-1 regulates } \\
\text { sarcomere formation and } \\
\text { suppresses smooth muscle } \\
\text { gene expression in the } \\
\text { mammalian heart }\end{array}$ & $\begin{array}{l}\text { SRP029956; http://www. } \\
\text { ncbi.nlm.nih.gov/ } \\
\text { sra/?term=SRP029956 }\end{array}$ & $\begin{array}{l}\text { Sequence Read } \\
\text { Archive (SRA). }\end{array}$ \\
\hline $\begin{array}{l}\text { Heidersbach A, Saxby C, } \\
\text { Carver-Moore K, Huang Y, } \\
\text { Ang Y-S, Ivey KN, } \\
\text { Srivastava D }\end{array}$ & 2013 & $\begin{array}{l}\text { Wildtype, miR-1-1 KO, miR-1 } \\
\text { Double het P2 mixed strain } \\
\text { heart analysis (MoGene } \\
2.0 \text { ST Arrays) }\end{array}$ & $\begin{array}{l}\text { GSE51394; http://www. } \\
\text { ncbi.nlm.nih.gov/geo/ } \\
\text { query/acc.cgi? } \\
\text { acc=GSE51394 }\end{array}$ & $\begin{array}{l}\text { Gene Expression } \\
\text { Omnibus (GEO). }\end{array}$ \\
\hline
\end{tabular}

\section{References}

Aoki HH, Sadoshima JJ, Izumo SS. 2000. Myosin light chain kinase mediates sarcomere organization during cardiac hypertrophy in vitro. Nat Med 6:183-8. doi: 10.1038/72287.

Bartel DP. 2009. MicroRNAs: target recognition and regulatory functions. Cell 136:215-33. doi: 10.1016/j. cell.2009.01.002.

Chen J, Kitchen CM, Streb JW, Miano JM. 2002. Myocardin: a component of a molecular switch for smooth muscle differentiation. J Mol Cell Cardiol 34:1345-56. doi: 10.1006/jmcc.2002.2086.

Chen JF, Mandel EM, Thomson JM, Wu Q, Callis TE, Hammond SM, et al. 2005. The role of microRNA-1 and microRNA-133 in skeletal muscle proliferation and differentiation. Nat Genet 38:228-33. doi: 10.1038/ng1725.

Choudhury N, Khromov AS, Somlyo AP, Somlyo AV. 2004. Telokin mediates Ca2+-desensitization through activation of myosin phosphatase in phasic and tonic smooth muscle. J Muscle Res Cell Motil 25:657-65. doi: 10.1007/s10974-004-7807-x.

Cordes KR, Sheehy NT, White MP, Berry EC, Morton SU, Muth AN, et al. 2009. miR-145 and miR-143 regulate smooth muscle cell fate and plasticity. Nature 460:705-10. doi: 10.1038/nature08195.

Cordes KR, Srivastava D. 2009. MicroRNA regulation of cardiovascular development. Circ Res 104:724-32. doi: 10.1161/CIRCRESAHA.108.192872.

Creemers EE, Sutherland LB, Oh J, Barbosa AC, Olson EN. 2006. Coactivation of MEF2 by the SAP domain proteins myocardin and MASTR. Mol Cell 23:83-96. doi: 10.1016/j.molcel.2006.05.026.

Davis JS, Hassanzadeh S, Winitsky S, Lin H, Satorius C, Vemuri R, et al. 2001. The overall pattern of cardiac contraction depends on a spatial gradient of myosin regulatory light chain phosphorylation. Cell 107:631-41. doi: 10.1016/S0092-8674(01)00586-4.

Dudnakova TV, Lakomkin VL, Tsyplenkova VG, Shekhonin BV, Shirinsky VP, Kapelko VI. 2003. Alterations in myocardial cytoskeletal and regulatory protein expression following a single Doxorubicin injection. $J$ Cardiovasc Pharmacol 41:788-94.

Fish JE, Santoro MM, Morton SU, Yu S, Yeh RF, Wythe JD, et al. 2008. miR-126 regulates angiogenic signaling and vascular integrity. Dev Cell 15:272-84. doi: 10.1016/j.devcel.2008.07.008.

Herring BP, El-Mounayri OO, Gallagher PJ, Yin FF, Zhou JJ. 2006. Regulation of myosin light chain kinase and telokin expression in smooth muscle tissues. Am J Physiol Cell Physiol 291:C817-27. doi: 10.1152/ajpcell.00198.2006.

Herring BP, Lyons GE, Hoggatt AM, Gallagher PJ. 2001. Telokin expression is restricted to smooth muscle tissues during mouse development. Am J Physiol Cell Physiol 280:C12-21.

Hom JR, Quintanilla RA, Hoffman DL, de Mesy Bentley KL, Molkentin JD, Sheu SS, et al. 2011. The permeability transition pore controls cardiac mitochondrial maturation and myocyte differentiation. Dev Cell 21:469-78. doi: 10.1016/j.devcel.2011.08.008.

Hoofnagle MH, Neppl RL, Berzin EL, Teg Pipes GC, Olson EN, Wamhoff BW, et al. 2011. Myocardin is differentially required for the development of smooth muscle cells and cardiomyocytes. Am J Physiol Heart Circ Physiol 300:H1707-21. doi: 10.1152/ajpheart.01192.2010.

Imamura M, Long X, Nanda V, Miano JM. 2010. Expression and functional activity of four myocardin isoforms. Gene 464:1-10. doi: 10.1016/j.gene.2010.03.012.

Ivey KN, Muth A, Arnold J, King FW, Yeh R-F, Fish JE, et al. 2008. MicroRNA regulation of cell lineages in mouse and human embryonic stem cells. Cell Stem Cell 2:219-29. doi: 10.1016/j.stem.2008.01.016.

Kamm KE, Stull JT. 2001. Dedicated myosin light chain kinases with diverse cellular functions. J Biol Chem 276:4527-30. doi: 10.1074/jbc.R000028200.

Khromov AS, Momotani KK, Jin LL, Artamonov MV, Shannon JJ, Eto MM, et al. 2012. Molecular mechanism of telokin-mediated disinhibition of myosin light chain phosphatase and cAMP/cGMP-induced relaxation of gastrointestinal smooth muscle. J Biol Chem 287:20975-85. doi: 10.1074/jbc.M112.341479.

Kim D, Pertea G, Trapnell C, Pimentel H, Kelley R, Salzberg SL. 2013. TopHat2: accurate alignment of transcriptomes in the presence of insertions, deletions and gene fusions. Genome Biol 14:R36. doi: 10.1186/gb-2013-14-4-r36.

King IN, Qian L, Liang J, Huang Y, Shieh JT, Kwon C, et al. 2011. A genome-wide screen reveals a role for microRNA-1 in modulating cardiac cell polarity. Dev Cell 20:497-510. doi: 10.1016/j.devcel.2011.03.010. 
Kwon C, Han Z, Olson EN, Srivastava D. 2005. MicroRNA1 influences cardiac differentiation in Drosophila and regulates Notch signaling. Proc Natl Acad Sci USA 102:18986-91. doi: 10.1073/pnas.0509535102.

Lee TI, Johnstone SE, Young RA. 2006. Chromatin immunoprecipitation and microarray-based analysis of protein location. Nat Protoc 1:729-48. doi: 10.1038/nprot.2006.98.

Li L, Liu Z, Mercer B, Overbeek P, Olson EN. 1997. Evidence for serum response factor-mediated regulatory networks governing SM22alpha transcription in smooth, skeletal, and cardiac muscle cells. Dev Biol 187:311-21. doi: 10.1006/ dbio.1997.8621.

Li S, Wang DZ, Wang Z, Richardson JA, Olson EN. 2003. The serum response factor coactivator myocardin is required for vascular smooth muscle development. Proc Natl Acad Sci USA 100:9366-70. doi: 10.1073/pnas.1233635100.

Liu N, Bezprozvannaya S, Williams AH, Qi X, Richardson JA, Bassel-Duby R, et al. 2008. microRNA-133a regulates cardiomyocyte proliferation and suppresses smooth muscle gene expression in the heart. Genes Dev 22:3242-54. doi: 10.1101/gad.1738708.

Liu N, Williams AH, Kim Y, McAnally J, Bezprozvannaya S, Sutherland LB, et al. 2007. An intragenic MEF2dependent enhancer directs muscle-specific expression of microRNAs 1 and 133. Proc Natl Acad Sci USA 104:20844-9. doi: 10.1073/pnas.0710558105.

McLean CY, Bristor D, Hiller M, Clarke SL, Schaar BT, Lowe CB, et al. 2010. GREAT improves functional interpretation of cis-regulatory regions. Nat Med 28:495-501. doi: 10.1038/nbt.1630.

Mishima Y, Abreu-Goodger C, Staton AA, Stahlhut C, Shou C, Cheng C, et al. 2009. Zebrafish miR-1 and miR-133 shape muscle gene expression and regulate sarcomeric actin organization. Genes Dev 23:619-32. doi: 10.1101/gad.1760209.

Niu Z, lyer D, Conway SJ, Martin JF, Ivey K, Srivastava D, et al. 2008. Serum response factor orchestrates nascent sarcomerogenesis and silences the biomineralization gene program in the heart. Proc Natl Acad Sci USA 105:17824-9. doi: 10.1073/pnas.0805491105.

Niu Z, Li A, Zhang SX, Schwartz RJ. 2007. Serum response factor micromanaging cardiogenesis. Curr Opin Cell Biol 19:618-27. doi: 10.1016/j.ceb.2007.09.013.

Poetter K, Jiang H, Hassanzadeh S, Master SR, Chang A, Dalakas MC, et al. 1996. Mutations in either the essential or regulatory light chains of myosin are associated with a rare myopathy in human heart and skeletal muscle. Nat Genet 13:63-9. doi: 10.1038/ng0596-63.

Qian L, van Laake LW, Huang Y, Liu S, Wendland MF, Srivastava D. 2011. miR-24 inhibits apoptosis and represses Bim in mouse cardiomyocytes. J Exp Med 208:549-60. doi: 10.1084/jem.20101547.

Rao PK, Kumar RM, Farkhondeh M, Baskerville S, Lodish HF. 2006. Myogenic factors that regulate expression of muscle-specific microRNAs. Proc Natl Acad Sci USA 103:8721-6. doi: 10.1073/pnas.0602831103.

Rao PK, Toyama Y, Chiang HR, Gupta S, Bauer M, Medvid R, et al. 2009. Loss of cardiac microRNA-mediated regulation leads to dilated cardiomyopathy and heart failure. Circ Res 105:585-94. doi: 10.1161/ CIRCRESAHA.109.200451.

Rottbauer W, Wessels G, Dahme T, Just S, Trano N, Hassel D, et al. 2006. Cardiac myosin light chain-2: a novel essential component of thick-myofilament assembly and contractility of the heart. Circ Res 99:323-31. doi: 10.1161/01.RES.0000234807.16034.fe.

Sepulveda JL, Vlahopoulos S, lyer D, Belaguli N, Schwartz RJ. 2002. Combinatorial expression of GATA4, Nkx2-5, and serum response factor directs early cardiac gene activity. J Biol Chem 277:25775-82. doi: 10.1074/jbc.M203122200.

Sokol NS, Ambros V. 2005. Mesodermally expressed Drosophila microRNA-1 is regulated by twist and is required in muscles during larval growth. Genes Dev 19:2343-54. doi: 10.1101/gad.1356105.

Srivastava D. 2006. Making or breaking the heart: from lineage determination to morphogenesis. Cell 126:1037-48. doi: 10.1016/j.cell.2006.09.003.

Thomas SS, Li XY, Sabo PJP, Sandstrom RR, Thurman RE, Canfield TK, et al. 2011. Dynamic reprogramming of chromatin accessibility during Drosophila embryo development. Genome Biol 12:R43. doi: 10.1186/ gb-2011-12-5-r43.

Treisman R. 1986. Identification of a protein-binding site that mediates transcriptional response of the c-fos gene to serum factors. Cell 46:567-74. doi: 10.1016/0092-8674(86)90882-2.

Treisman R. 1987. Identification and purification of a polypeptide that binds to the c-fos serum response element. EMBO J 6:2711-7.

Valencia-Sanchez MA, Liu J, Hannon GJ, Parker R. 2006. Control of translation and mRNA degradation by miRNAs and siRNAs. Genes Dev 20:515-24. doi: 10.1101/gad.1399806.

Wang D, Chang PS, Wang Z, Sutherland L, Richardson JA, Small E, et al. 2001. Activation of cardiac gene expression by myocardin, a transcriptional cofactor for serum response factor. Cell 105:851-62. doi: 10.1016/ S0092-8674(01)00404-4.

Wang DZ, Olson EN. 2004. Control of smooth muscle development by the myocardin family of transcriptional coactivators. Curr Opin Genet Dev 14:558-66. doi: 10.1016/j.gde.2004.08.003.

Wang Z, Wang D-Z, Pipes GCT, Olson EN. 2003. Myocardin is a master regulator of smooth muscle gene expression. Proc Natl Acad Sci USA 100:7129-34. doi: 10.1073/pnas.1232341100.

Zambon AC, Gaj S, Ho I, Hanspers K, Vranizan K, Evelo CT, et al. 2012. GO-Elite: a flexible solution for pathway and ontology over-representation. Bioinformatics 28:2209-10. doi: 10.1093/bioinformatics/bts366.

Zhao Y, Ransom JF, Li A, Vedantham V, Drehle von M, Muth AN, et al. 2007. Dysregulation of cardiogenesis, cardiac conduction, and cell cycle in mice lacking miRNA-1-2. Cell 129:303-17. doi: 10.1016/j.cell.2007.03.030.

Zhao Y, Samal E, Srivastava D. 2005. Serum response factor regulates a muscle-specific microRNA that targets Hand2 during cardiogenesis. Nature Publishing Group 436:214-20. doi: 10.1038/nature03817. 